Supporting Information

\title{
Recent advances in sulfidated zerovalent iron for contaminant transformation
}

\author{
Ariel Nunez Garcia, ${ }^{1,+}$ Yanyan Zhang, ${ }^{2,3,+}$ Subhasis Ghoshal, ${ }^{2}$ Feng He, ${ }^{4}$ Denis M. O'Carroll ${ }^{* 5}$ \\ ${ }^{1}$ Department of Civil and Environmental Engineering, \\ Western University, 1151 Richmond Rd., London, ON, N6A 5B8, Canada \\ ${ }^{2}$ Department of Civil Engineering, \\ McGill University, 817 Sherbrooke Street West, Montreal, QC, H3A 0C3, Canada \\ ${ }^{3}$ Key Laboratory of Coastal Environment and Resources of Zhejiang Province, \\ School of Engineering, Westlake University, Hangzhou 310024, Zhejiang Province, China. \\ ${ }^{4}$ Institute of Environmental Chemistry and Pollution Control \\ College of Environment, Zhejiang University of Technology \\ 18 Chaowang Rd, Hangzhou, China 310014 \\ ${ }^{5}$ School of Civil and Environmental Engineering, Water Research Centre, \\ University of New South Wales, Sydney NSW 2052, Australia \\ \$These authors contributed equally to this work
}

Number of pages: 21

Number of figures: 2

Number of tables: 6

\section{*Corresponding author}

School of Civil \& Environmental Engineering

Civil Engineering Building (H20), Room CE303

Kensington Campus, University of New South Wales

Sydney, NSW 2052 Australia

Phone: (+61 2) 80719822

Email: d.ocarroll@unsw.edu.au 


\section{Table of Contents:}

Table S1. Summary of the Data on the Degradation of Trichloroethene (TCE) by S-(n)ZVI under Anoxic 41 and Stoichiometrically $\mathrm{Fe}^{0}$ Excess Conditions.

42 Table S2. Summary of the Data on the Degradation of TCE by S-(n)ZVI under Anoxic and 43 Stoichiometrically $\mathrm{Fe}^{0}$ Limited Conditions.

44 Table S3. Summary of the Data on the Degradation of Chlorinated Hydrocarbons (CHCs) other than TCE 45 by S-(n)ZVI under Anoxic and Stoichiometrically Fe ${ }^{0}$ Excess Conditions.

46 Table S4. Summary of the Data on the Degradation of Contaminants (Nonmetal) other than Chlorinated 47 Hydrocarbons (CHCs) by S-(n)ZVI under Anoxic Conditions.

48 Table S5. Summary of the Data on the Degradation of Contaminants by S-(n)ZVI under Oxic Conditions.

49 Table S6. Summary of the Data on the Degradation of Metals, Metalloids, and Oxyanions by S-(n)ZVI.

$50 \quad$ Figure S1. Number of peer-reviewed journal papers on S-(n)ZVI from 2011.

51 Figure S2. Correlation of the enhancement ratio $(R)$ in reactivity of TCE induced by sulfidation of (n)ZVI

52 with the $[\mathrm{S} / \mathrm{Fe}]_{\text {dosed }}$ of $\mathrm{S}-(\mathrm{n}) \mathrm{ZVI}$ synthesized by (A) two-step method with $\mathrm{Na}_{2} \mathrm{~S}$ and (B) one-step method

53 with $\mathrm{Na}_{2} \mathrm{~S}_{2} \mathrm{O}_{4}$. The correlation coefficient $(r)$ and the significance level $(p)$ are shown. 


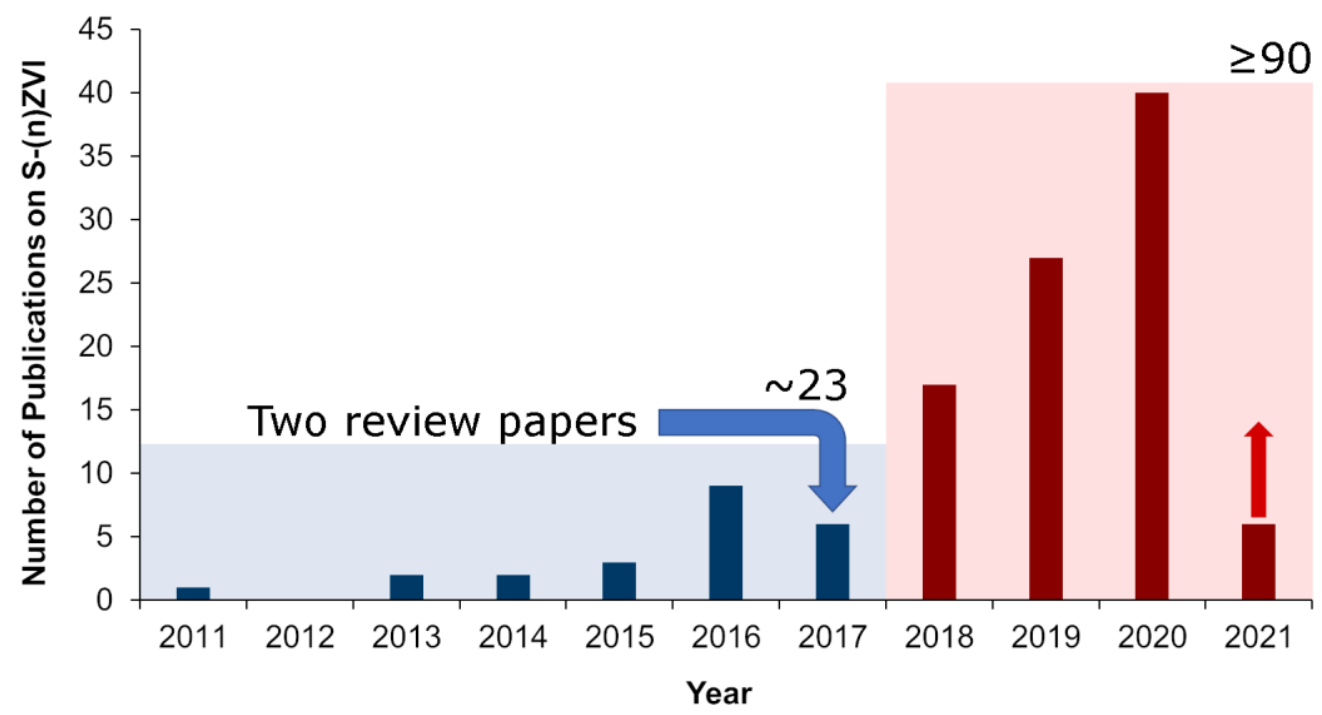

Figure S1. Number of peer-reviewed journal papers on S-(n)ZVI from 2011. 


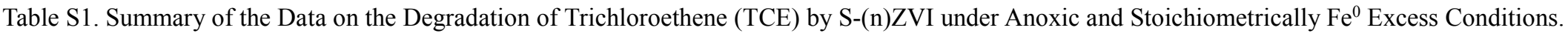

\begin{tabular}{|c|c|c|c|c|c|c|c|c|}
\hline Target & Sulfidation method & $\begin{array}{l}\text { Optimal } \\
\text { S/Fe }\end{array}$ & $\begin{array}{c}\text { HER, } \\
k \mathrm{~s}-(\mathrm{n}) \mathrm{ZVI} / k_{(\mathrm{n}) \mathrm{ZVI}}, \\
\mu \mathrm{mol} \cdot \mathrm{L} \cdot \mathrm{g}^{-1} .\end{array}$ & $\begin{array}{c}\text { Contaminant } \\
k \mathrm{~s} \text {-(n)ZVII } k_{(\mathrm{n}) \mathrm{ZVI}}, \\
\mathbf{L} \cdot \mathrm{g}^{-1} \cdot \mathbf{h}^{-1}\end{array}$ & Products & Influencing factors & Proposed mechanisms* & References \\
\hline \multicolumn{9}{|c|}{ S-(n)ZVI, anoxic, $\mathrm{Fe}^{0}$ excess, TCE } \\
\hline TCE & $\begin{array}{l}\text { mZVI+ NaHS, two- } \\
\text { step }\end{array}$ & Dosed 0.011 & n.a. & $\begin{array}{c}\text { n.a. } \\
\text { No degradation } \\
\text { by mZVI }\end{array}$ & $\begin{array}{c}\text { Ethene, acetylene, } \\
\text { ethane, cis-DCE, trans- } \\
\text { DCE }\end{array}$ & n.a. & $\begin{array}{l}\text { Increased electrical conductivity; } \\
\mathrm{H}_{2} \text { helps the dechlorination by } \\
\text { FeS }\end{array}$ & (Hassan, 2000) \\
\hline TCE & $\begin{array}{l}\text { mZVI + NaHS, two- } \\
\text { step }\end{array}$ & $\begin{array}{l}\text { Dosed } \\
0.00056\end{array}$ & n.a. & 2 & $\begin{array}{c}\text { Ethene, acetylene, } \\
\text { ethane, cis-DCE, } 1,1- \\
\text { DCE }\end{array}$ & n.a. & $\begin{array}{l}\text { Increased electrical conductivity; } \\
\text { the deposit FeS has higher } \\
\text { reactivity for TCE }\end{array}$ & (Butler and Hayes, 2001) \\
\hline TCE & $\begin{array}{l}\mathrm{Fe}^{3+}+\underset{\mathrm{Na}_{2} \mathrm{~S}_{2} \mathrm{O}_{4}, \text { one- }}{\text { step }} \\
\end{array}$ & Dosed 0.14 & n.a. & 21.3 & $\begin{array}{l}\text { Small amount of cis- } \\
\text { DCE as intermediates; } \\
\text { others not indicated }\end{array}$ & n.a. & $\begin{array}{l}\text { Increased surface area and } \\
\text { electrical conductivity }\end{array}$ & (Kim et al., 2011) \\
\hline TCE & $\begin{array}{l}\mathrm{Fe}^{3+}+\underset{\mathrm{Na}_{2} \mathrm{~S}_{2} \mathrm{O}_{4}, \text { one- }}{\text { step }} \\
\end{array}$ & Dosed 0.20 & n.a. & n.a. & n.a. & $\begin{array}{c}\text { Not affected by IS; increased } \\
\text { with } \mathrm{pH}, \mathrm{Ca}^{2+}, \mathrm{Mg}^{2+} ; \text { reduced by } \\
\mathrm{HA} \text { and in } \mathrm{GW}\end{array}$ & $\begin{array}{c}\text { Greater driving force of } \\
\text { deprotonated ligands in electron } \\
\text { donation }\end{array}$ & (Kim et al., 2013) \\
\hline TCE & $\begin{array}{l}\mathrm{Fe}^{3+}+\mathrm{Na}_{2} \mathrm{~S}_{2} \mathrm{O}_{4} \text {, one- } \\
\text { step }\end{array}$ & n.a. & n.a. & n.a. & Ethane; butane & $\begin{array}{l}\text { Increased with } \mathrm{Co}^{2+}, \mathrm{Ni}^{2+}, \mathrm{Pd}^{2+} ; \\
\text { reduced with } \mathrm{Mn}^{2+}, \mathrm{Gu}^{2+}\end{array}$ & n.a. & (Kim et al., 2014) \\
\hline TCE & $\begin{array}{l}\mathrm{nZVI}+\mathrm{Na}_{2} \mathrm{~S}, \text { two- } \\
\text { step }\end{array}$ & Dosed 0.045 & 0.4 & 37.8 & $\begin{array}{l}\text { Ethene; acetylene; } \\
\text { ethane }\end{array}$ & $\begin{array}{l}\text { Increased with } \mathrm{pH}(7.0,9.0 . \\
\text { 11.0); slightly reduced in } \mathrm{GW} \\
\text { not affected by } \mathrm{CMC}\end{array}$ & $\begin{array}{l}\text { Increased electrical conductivity } \\
\text { and hydrophobicity }\end{array}$ & $\begin{array}{c}\text { (Rajajayavel and Ghoshal, } \\
\text { 2015) }\end{array}$ \\
\hline TCE & $\begin{array}{l}\text { nZVI }+\mathrm{Na}_{2} \mathrm{~S}_{2} \mathrm{O}_{3} \\
\text { two-step }\end{array}$ & Dosed 0.05 & 0.23 & 34.8 & $\begin{array}{l}\text { Ethene; ethane; } \\
\text { C3-C6; acetylene as } \\
\text { intermediates }\end{array}$ & n.a. & $\begin{array}{l}\text { Poisoning of } \mathrm{H}^{*} \text { recombination } \\
\text { by sulfur }\end{array}$ & (Han and Yan, 2016) \\
\hline TCE & $\begin{array}{l}\mathrm{CMC}-\mathrm{nZVI}, \\
\mathrm{Na}_{2} \mathrm{~S}_{2} \mathrm{O}_{4} \text { or } \mathrm{Na}_{2} \mathrm{~S} \\
\text { two-step }\end{array}$ & $\begin{array}{l}\text { Dosed } 0.19 \\
\quad \text { or } 1\end{array}$ & Negligible & 1.4 & Ethene & Reduced after aging & $\begin{array}{l}\text { Sulfidation eliminates reaction } \\
\text { with water }\end{array}$ & (Fan et al., 2016) \\
\hline TCE & $\begin{array}{l}\text { mZVI }+\mathrm{S}^{0}, \text { ball } \\
\text { milling }\end{array}$ & $\begin{array}{l}\text { Dosed } 0.1 ; \\
\text { particle } 0.1\end{array}$ & 0.19 & 36.8 & $\begin{array}{l}\text { Acetylene, ethene, } \\
\text { ethane, cis-DCE, } \\
\text { C3-C6 }\end{array}$ & n.a. & $\begin{array}{c}\text { Reduced reaction with water; } \\
\text { increased electrical conductivity }\end{array}$ & (Gu et al., 2017) \\
\hline TCE & $\begin{array}{l}\text { nZVI }+\mathrm{Na}_{2} S, \text { two- } \\
\text { step }\end{array}$ & $\begin{array}{l}\text { Dosed } 0.021 ; \\
\text { particle } 0.014\end{array}$ & n.a. & 11 & $\begin{array}{l}\text { Acetylene, ethene, } \\
\text { ethane }\end{array}$ & Reduced by HA and rhamnolipid & n.a. & $\begin{array}{l}\text { (Bhattacharjee and } \\
\text { Ghoshal, 2018a) }\end{array}$ \\
\hline TCE & $\begin{array}{l}\mathrm{nZVI}+\mathrm{Na}_{2} \mathrm{~S}, \text { two- } \\
\text { step }\end{array}$ & $\begin{array}{c}\text { Dosed } 0.1 ; \\
\text { particle } 0.042\end{array}$ & 0.44 & 41 & n.a. & n.a. & $\begin{array}{l}\text { Increased electrical conductivity } \\
\text { and hydrophobicity }\end{array}$ & $\begin{array}{l}\text { (Bhattacharjee and } \\
\text { Ghoshal, 2018b) }\end{array}$ \\
\hline TCE & $\begin{array}{l}\mathrm{Fe}^{2+}+\mathrm{Na}_{2} \mathrm{~S}, \text { one- } \\
\text { step }\end{array}$ & $\begin{array}{l}\text { Dosed } 0.4 \\
\text { particle } 0.4\end{array}$ & 2.04 & 106 & n.a. & n.a. & $\begin{array}{l}\text { Increased electrical conductivity } \\
\text { and hydrophobicity }\end{array}$ & \\
\hline TCE & $\begin{array}{l}\mathrm{Fe}^{3+}+\underset{\text { step }}{\mathrm{Na}_{2} \mathrm{~S}_{2} \mathrm{O}_{4} \text {, one- }} \\
\text { s. }\end{array}$ & Dosed 0.017 & n.a. & n.a. & n.a. & $\begin{array}{c}\text { Increased with } \mathrm{pH} ; \text { not much } \\
\text { affected by DO and aging for } 20 \\
\text { days }\end{array}$ & n.a. & (Dong et al., 2018) \\
\hline TCE & $\begin{array}{l}\text { nZVI + } \\
\text { step }\end{array}$ & Dosed 0.067 & n.a. & 7 & n.a. & $\begin{array}{l}\text { Reduced by NOM, GW, and DO; } \\
\text { increased with } \mathrm{pH} \text { and } \\
\text { temperature }\end{array}$ & n.a. & (Han et al., 2019) \\
\hline TCE & $\begin{array}{l}\mathrm{Fe}^{2+}+\underset{\mathrm{Na}_{2} \mathrm{~S}_{2} \mathrm{O}_{4} \text {, one- }}{\text { step }} \\
\end{array}$ & Dosed 0.1 & n.a. & 11.8 & $\begin{array}{l}10 \% \text { cis-DCE as } \\
\text { intermediates; other } \\
\text { products not indicated }\end{array}$ & Reduced with aging in $\mathrm{GW}$ & $\begin{array}{l}\text { FeS increases TCE degradation } \\
\text { via direct electron transfer }\end{array}$ & $\begin{array}{c}\text { (Mangayayam et al., } \\
2019 \mathrm{a})\end{array}$ \\
\hline
\end{tabular}




\begin{tabular}{|c|c|c|c|c|c|c|c|c|}
\hline TCE & $\begin{array}{l}\text { nZVI + } \mathrm{Na}_{2} \mathrm{~S}, \text { two- } \\
\text { step }\end{array}$ & Dosed 0.23 & n.a. & n.a. & n.a. & $\begin{array}{l}\text { Reduced to } 59 \% \text { of the initial } \\
\text { degradation with aging in GW for } \\
120 \text { days }\end{array}$ & n.a. & $\begin{array}{l}\text { (Mangayayam et al., } \\
2019 \mathrm{~b})\end{array}$ \\
\hline TCE & $\begin{array}{c}\mathrm{Fe}^{3+}+\underset{\text { Nap }}{\mathrm{Na}_{2} \mathrm{~S}_{2} \mathrm{O}_{4} \text {, one- }} \\
\text { step }\end{array}$ & $\begin{array}{c}\text { Dosed } 0.14 \\
\text { particle } 0.054\end{array}$ & 0.03 & 8.8 & $\begin{array}{l}\text { Ethene, ethane; } \\
\text { acetylene as } \\
\text { intermediate }\end{array}$ & $\begin{array}{l}\text { Reduced with aging in water for } \\
60 \text { days }\end{array}$ & Increased hydrophobicity & (Xu et al., 2019a) \\
\hline TCE & $\begin{array}{l}\text { nZVI + } \mathrm{Na}_{2} \mathrm{~S}, \text { two- } \\
\text { step }\end{array}$ & $\begin{array}{c}\text { Dosed } 0.14 ; \\
\text { particle } 0.008\end{array}$ & 0.17 & 7.9 & $\begin{array}{l}\text { Ethene, ethane; } \\
\text { acetylene as } \\
\text { intermediate }\end{array}$ & $\begin{array}{l}\text { Reduced with aging in water for } \\
60 \text { days }\end{array}$ & Increased hydrophobicity & \\
\hline TCE & $\begin{array}{l}\text { nZVI + } \\
\text { step }\end{array}$ & $\begin{array}{l}\text { Dosed 0.1; } \\
\text { Particle } \\
0.0065\end{array}$ & 0.46 & 14 & $\begin{array}{l}\text { Ethene, ethane, } \\
\text { acetylene }\end{array}$ & n.a. & Increased electrical conductivity & (Xu et al., 2019b) \\
\hline TCE & $\begin{array}{l}\text { mZVI }+\mathrm{S}^{0} \text {, ball } \\
\text { milling }\end{array}$ & Dosed 0.1 & $\begin{array}{l}0.04-0.29(\mathrm{pH} \\
6-8) ; 8,7(\mathrm{pH} \\
9) ; 208(\mathrm{pH} 10)\end{array}$ & $\begin{array}{l}0.41(\mathrm{pH} 6) \\
14-160(\mathrm{pH} \\
7-10)\end{array}$ & $\begin{array}{l}\text { Methane, acetylene, } \\
\text { ethene, ethane, cis- } \\
\text { DCE, 1,1-DCE, C3-C6 }\end{array}$ & $\begin{array}{l}\text { Optimal at } \mathrm{pH} 6 \text {; decrease with } \\
\text { increasing } \mathrm{pH}\end{array}$ & $\begin{array}{c}\mathrm{S} \text { weaken the interactions of ZVI } \\
\text { with } \mathrm{H}_{2} \mathrm{O} \text { molecule (DFT } \\
\text { calculations) }\end{array}$ & (Gu et al., 2019) \\
\hline TCE & $\begin{array}{l}\text { mZVI }+\mathrm{S}^{0} \text {, ball } \\
\text { milling }\end{array}$ & Dosed 0.1 & n.a. & n.a. & n.a. & Dramatically reduced by $\mathrm{Cr}(\mathrm{VI})$ & n.a. & (Zou et al., 2019) \\
\hline TCE & $\begin{array}{c}\mathrm{Fe}^{3+}+\underset{\text { Nap }}{\mathrm{Na}_{2} \mathrm{~S}_{2} \mathrm{O}_{4} \text {, one- }} \\
\text { step }\end{array}$ & Dosed 0.02 & n.a. & $\begin{array}{c}\text { No data on } \\
\text { nZVI }\end{array}$ & n.a. & $\begin{array}{l}\text { Reduced by } \mathrm{Cr}^{6+}, \mathrm{Cd}^{2+} \text {; slightly } \\
\text { increased by } \mathrm{NO}_{3}^{-}\end{array}$ & n.a. & (Wang et al., 2020) \\
\hline TCE & $\begin{array}{l}\mathrm{mZVI}+\mathrm{Na}_{2} \mathrm{~S}_{2} \mathrm{O}_{3} \\
\text { two-step }\end{array}$ & Dosed 0.05 & n.a. & $\begin{array}{c}\text { Highly } \\
\text { improved; no } \\
\text { degradation by } \\
\text { mZVI }\end{array}$ & Ethene, ethane, C3-C6 & $\begin{array}{l}\text { Reduced by Cu-amendment and } \\
\text { further reduced with following } \\
\text { sulfidation }\end{array}$ & n.a. & (Islam et al., 2020) \\
\hline TCE & $\begin{array}{c}\mathrm{CMC}, \mathrm{Fe}^{2+}+ \\
\mathrm{Na}_{2} \mathrm{~S}_{2} \mathrm{O}_{4}, \text { one-step }\end{array}$ & Dosed 0.05 & Reduced & 7.4 & $\begin{array}{l}\text { Ethene, ethane, } \\
\text { acetylene, } \mathrm{C} 3-\mathrm{C} 6\end{array}$ & $\begin{array}{c}\text { Increased by stabilization with } \\
\text { CMC }\end{array}$ & n.a. & (Xu et al., 2020c) \\
\hline TCE & $\begin{array}{l}\text { CMC-nZVI, } \mathrm{Na}_{2} \mathrm{~S}, \\
\text { two-step }\end{array}$ & Dosed 0.2 & Reduced & 67.5 & $\begin{array}{l}\text { Ethene, ethane, } \\
\text { acetylene, C3-C6 }\end{array}$ & $\begin{array}{l}\text { Increased by stabilization with } \\
\text { CMC }\end{array}$ & n.a. & \\
\hline TCE & $\begin{array}{l}\text { nZVI + } \\
\text { step } \\
\text { sta }\end{array}$ & Dosed 0.23 & n.a. & n.a. & n.a. & $\begin{array}{c}\text { Reduced to } 55 \% \text { after aging in } \\
\text { water for } 7 \text { days; the reduction is } \\
\text { counterbalanced by } \mathrm{Cl}^{-}, \mathrm{HCO}_{3}^{-}, \\
\mathrm{CO}_{3}^{2-} \text { and further lowered by } \\
\mathrm{SO}_{4}{ }^{2-}, \mathrm{Ca}^{2+}, \text { and } \mathrm{HPO}_{4}{ }^{2-}\end{array}$ & n.a. & (Mangayayam et al., 2020) \\
\hline TCE & $\begin{array}{c}\mathrm{Fe}^{3+}+\mathrm{Na}_{2} \mathrm{~S}_{2} \mathrm{O}_{4}, \text { one- } \\
\text { step }\end{array}$ & Dosed 0.05 & n.a. & 3.0 & Acetylene & $\begin{array}{l}\text { Increased with graphene aerogel } \\
\text { and with increasing } \mathrm{pH} \text { and } \\
\text { temperature }\end{array}$ & Increased electrical conductivity & (Bin et al., 2020) \\
\hline TCE & $\begin{array}{c}\mathrm{Fe}^{3+}+ \\
\text { montmorillonite }+ \\
\mathrm{Na}_{2} \mathrm{~S} \text {, one-step }\end{array}$ & Dosed 1 & n.a. & 1.8 & $\begin{array}{l}\text { Ethene, acetylene, } \\
\text { C3-C6, chloride }\end{array}$ & $\begin{array}{l}\text { Not affected by HA and aging in } \\
\text { water for } 30 \text { days; increased with } \\
\text { increasing initial pH }\end{array}$ & n.a. & (Xu et al., 2020a) \\
\hline TCE & $\begin{array}{c}\mathrm{Fe}^{3+}+\underset{\text { step }}{\mathrm{Na}_{2} \mathrm{~S}_{2} \mathrm{O}_{4}, \text { one- }} \\
\text { ste }\end{array}$ & $\begin{array}{l}\text { Dosed } 0.035 \\
\text { particle } 0.01\end{array}$ & 6.35 & 50 & $\begin{array}{l}\text { Ethene, ethane, } \\
\text { acetylene }\end{array}$ & n.a. & $\begin{array}{l}\text { Reduced electron resistance; } \\
\text { increased hydrophobicity }\end{array}$ & (Xu et al., 2020b) \\
\hline TCE & $\begin{array}{c}\text { Commercial nZVI + } \\
\mathrm{Na}_{2} \mathrm{~S} \text {, two-step }\end{array}$ & $\begin{array}{l}\text { Dosed } 0.087 \\
\text { particle } 0.034\end{array}$ & Reduced & 12 & $\begin{array}{l}\text { Ethene, ethane, } \\
\text { acetylene }\end{array}$ & n.a. & $\begin{array}{l}\text { Increased electrical conductivity; } \\
\text { increased hydrophobicity }\end{array}$ & (Brumovský et al., 2020) \\
\hline TCE & $\begin{array}{l}\mathrm{Fe}^{2+}+\underset{\text { step }}{\mathrm{Na}_{2} \mathrm{~S}_{2} \mathrm{O}_{4} \text {, one- }} \\
\text { ste }\end{array}$ & $\begin{array}{c}\text { Dosed } 0.14 ; \\
\text { particle } 0.052\end{array}$ & 0.083 & 3.3 & $\begin{array}{l}\text { Ethene, ethane, } \\
\text { acetylene }\end{array}$ & n.a. & $\begin{array}{l}\text { Increased electrical conductivity; } \\
\text { increased hydrophobicity }\end{array}$ & (Xu et al., 2020d) \\
\hline
\end{tabular}




\begin{tabular}{|c|c|c|c|c|c|c|c|c|}
\hline TCE & $\begin{array}{c}\mathrm{Fe}^{3+}+\underset{\mathrm{Na}_{2} \mathrm{~S}_{2} \mathrm{O}_{4}, \text { one- }}{\text { step }} \\
\end{array}$ & $\begin{array}{c}\text { Dosed 0.14; } \\
\text { particle } 0.049\end{array}$ & 0.0015 & 14 & $\begin{array}{l}\text { Ethene, ethane, } \\
\text { acetylene }\end{array}$ & n.a. & $\begin{array}{l}\text { Increased electrical conductivity; } \\
\text { increased hydrophobicity }\end{array}$ & \\
\hline TCE & $\begin{array}{l}\mathrm{Fe}^{3+}+\mathrm{Na}_{2} \mathrm{~S} \text {, one- } \\
\text { step }\end{array}$ & $\begin{array}{l}\text { Dosed } 0.14 \\
\text { particle } 0.051\end{array}$ & 0.0028 & 35 & $\begin{array}{l}\text { Ethene, ethane, } \\
\text { acetylene }\end{array}$ & n.a. & $\begin{array}{l}\text { Increased electrical conductivity; } \\
\text { increased hydrophobicity }\end{array}$ & \\
\hline TCE & $\mathrm{Fe}^{3+}+\mathrm{K}_{2} \mathrm{~S}_{6}$, one-step & $\begin{array}{l}\text { Dosed } 0.14 ; \\
\text { particle } 0.04\end{array}$ & 0.12 & 5.7 & $\begin{array}{l}\text { Ethene, ethane, } \\
\text { acetylene }\end{array}$ & n.a. & $\begin{array}{l}\text { Increased electrical conductivity; } \\
\text { increased hydrophobicity }\end{array}$ & \\
\hline TCE & $\begin{array}{l}\mathrm{mZVI}+\mathrm{S}^{0} \text {, ball } \\
\text { milling }\end{array}$ & $\begin{array}{l}\text { Dosed } 0.1 ; \\
\text { particle } 0.1\end{array}$ & 0.31 & 8.8 & $\begin{array}{c}\text { methane, acetylene, } \\
\text { ethene, ethane, } \\
\text { cisDCE, 1,1-DCE, } \\
\text { C3-C6 }\end{array}$ & $\begin{array}{c}\text { Slightly enhanced by } \mathrm{PO}_{4}{ }^{3-} ; \text { not } \\
\text { much affected by } \mathrm{HCO}_{3}{ }^{-}, \mathrm{Cl}^{-}, \\
\mathrm{SO}_{4}{ }^{-{ }^{-}}\end{array}$ & n.a. & (Gong et al., 2020b) \\
\hline TCE & $\begin{array}{c}\text { Commercial mZVI + } \\
\mathrm{S}^{0} \text {, two-step }\end{array}$ & $\begin{array}{l}\text { Dosed } 0.1 \\
\text { particle } 0.1\end{array}$ & Reduced & 800 & n.a. & n.a. & n.a. & (Cai et al., 2021) \\
\hline TCE & $\begin{array}{c}\mathrm{mZVI}+\mathrm{S}^{0} \text {, ball } \\
\text { milling }\end{array}$ & $\begin{array}{l}\text { Dosed 0.1; } \\
\text { particle 0.1 }\end{array}$ & 0.21 & 10.6 & $\begin{array}{c}\text { methane, acetylene, } \\
\text { ethene, ethane, } \\
\text { cisDCE, 1,1-DCE, } \\
\text { C3-C6 }\end{array}$ & Suppressed by $\mathrm{NO}_{3}^{-}$ & n.a. & (Gong et al., 2021) \\
\hline
\end{tabular}

* The proposed mechanism for enhanced reactivity of S-(n)ZVI to (n)ZVI.

n.a. not available; HA, humic acid; GW, groundwater; IS, ionic strength; OM, organic matter; DO, dissolved oxygen; CMC, carboxymethyl cellulose; DFT, density functional theory.

Table S2. Summary of the Data on the Degradation of TCE by S-(n)ZVI under Anoxic and Stoichiometrically $\mathrm{Fe}^{0} \mathrm{Limited}_{\text {Conditions. }}$

\begin{tabular}{|c|c|c|c|c|c|c|c|c|}
\hline Target & $\begin{array}{l}\text { Sulfidation } \\
\text { method }\end{array}$ & $\begin{array}{l}\text { Optimal } \\
\text { S/Fe }\end{array}$ & $\begin{array}{c}\text { HER, } \\
k_{\text {S-(n)ZvI } k_{(\mathrm{n} Z Z \mathrm{ZVI}},} \\
\mu \mathrm{mol} \cdot \mathbf{L} \cdot \mathbf{g}^{-1}\end{array}$ & $\begin{array}{c}\text { Contaminant } \\
k_{\mathrm{S}-(\mathrm{n}) \mathrm{ZV}} \boldsymbol{k} k_{(\mathrm{n}) \mathrm{ZVI}}, \\
\mathbf{L} \cdot \mathbf{g}^{-1} \cdot \mathbf{h}^{-1}\end{array}$ & $\begin{array}{c}\text { Degradation } \\
\text { capacity, } \\
\mathrm{mol} / \mathrm{mol} \mathrm{Fe}^{0}\end{array}$ & Products & Proposed mechanisms* & References \\
\hline \multicolumn{9}{|c|}{ S-(n)ZVI, anoxic, $\mathrm{Fe}^{0}$ limited, CHCs } \\
\hline TCE & $\begin{array}{l}\text { mZVI }+\mathrm{S}^{0} \text {, ball } \\
\text { milling }\end{array}$ & Dosed 0.1 & 0.19 & 13 & 0.39 & $\begin{array}{l}\text { Acetylene, ethene, ethane, cis- } \\
\text { DCE, C3-C6 }\end{array}$ & $\begin{array}{l}\text { Reduced reaction with water; increased } \\
\text { electrical conductivity }\end{array}$ & (Gu et al., 2017) \\
\hline TCE & $\begin{array}{l}\text { nZVI }+\mathrm{Na}_{2} \mathrm{~S} \\
\text { two-step }\end{array}$ & Dosed 0.1 & 0.44 & 2.7 & 0.28 & Acetylene & $\begin{array}{l}\text { Increased electrical conductivity and } \\
\text { hydrophobicity }\end{array}$ & \multirow[t]{2}{*}{$\begin{array}{c}\text { (Bhattacharjee and } \\
\text { Ghoshal, 2018b) }\end{array}$} \\
\hline TCE & $\begin{array}{l}\mathrm{Fe}^{2+}+\mathrm{Na}_{2} \mathrm{~S} \\
\text { one-step }\end{array}$ & Dosed 0.4 & 2.04 & 8.9 & 0.46 & Acetylene & $\begin{array}{c}\text { Increased electrical conductivity and } \\
\text { hydrophobicity; FeS embedded in the particle }\end{array}$ & \\
\hline TCE & $\begin{array}{l}\text { nZVI }+\mathrm{Na}_{2} \mathrm{~S} \\
\text { two-step }\end{array}$ & Dosed 0.2 & 0.96 & $\begin{array}{c}\text { Highly improved; } \\
\text { almost no degradation } \\
\text { by nZVI }\end{array}$ & 0.32 & $\begin{array}{l}\text { Acetylene, ethene, ethane, } \\
\text { C3-C6 }\end{array}$ & $\begin{array}{c}\text { Increased electrical conductivity and FeS } \\
\text { conducting major electrons to TCE } \\
\text { dechlorination }\end{array}$ & (He et al., 2018) \\
\hline TCE & $\begin{array}{c}\text { nZVI }+\mathrm{Na}_{2} \mathrm{~S} \\
\text { two-step }\end{array}$ & $\begin{array}{l}\text { Dosed 0.1; } \\
\text { Particle } \\
0.0065\end{array}$ & 0.46 & 10 & n.a. & Ethene, acetylene & Increased electrical conductivity & (Xu et al., 2019b) \\
\hline
\end{tabular}


Table S3. Summary of the Data on the Degradation of Chlorinated Hydrocarbons (CHCs) other than TCE by S-(n)ZVI under Anoxic and Stoichiometrically Fe ${ }^{0}$ Excess Conditions.

\begin{tabular}{|c|c|c|c|c|c|c|c|c|}
\hline Target & $\begin{array}{c}\text { Sulfidation } \\
\text { method }\end{array}$ & $\begin{array}{l}\text { Optimal } \\
\text { S/Fe }\end{array}$ & 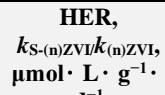 & $\begin{array}{c}\text { Contaminant } \\
k_{\mathrm{S}-(\mathrm{n}) \mathrm{ZVI} I} \boldsymbol{k}_{(\mathrm{n}) \mathrm{ZVI}} \\
\mathbf{L} \cdot \mathrm{g}^{-1} \cdot \mathbf{h}^{-1}\end{array}$ & Products & Influencing factors & Proposed mechanisms* & References \\
\hline \multicolumn{9}{|c|}{$\mathrm{S}$-(n)ZVI, anoxic, $\mathrm{Fe}^{0}$ excess, CHCs } \\
\hline PCE & $\begin{array}{l}\text { mZVI+ NaHS, } \\
\text { two-step }\end{array}$ & Dosed 0.011 & n.a. & $\begin{array}{c}\text { n.a. } \\
\text { No degradation by } \\
\text { mZVI }\end{array}$ & $\begin{array}{c}\text { Ethene, acetylene, } \\
\text { ethane, cis-DCE, trans- } \\
\text { DCE }\end{array}$ & n.a. & $\begin{array}{c}\text { Increased electrical } \\
\text { conductivity; } \mathrm{H}_{2} \text { helps the } \\
\text { dechlorination by FeS }\end{array}$ & (Hassan, 2000) \\
\hline PCE & $\begin{array}{l}\text { Carbo-nZVI + S } \\
\text { species }\end{array}$ & Dosed 0.11 & 0.006 & 3.2 & $\begin{array}{l}\text { Ethene, acetylene, } \\
\text { ethane }\end{array}$ & Increased slightly by $\mathrm{HCO}_{3}^{-}$ & n.a. & (Vogel et al., 2019) \\
\hline 1,2-DCA & $\begin{array}{l}\mathrm{nZVI}+\mathrm{Na}_{2} \mathrm{~S}_{2} \mathrm{O}_{4} \\
\text { two-step }\end{array}$ & Dosed 0.25 & n.a. & $\begin{array}{c}\text { n.a. } \\
\text { No degradation by } \\
\text { nZVI }\end{array}$ & $\beta$-Elimination & Small amount of ethene & n.a. & (Garcia et al., 2016) \\
\hline $1,1,1-\mathrm{TCA}$ & $\begin{array}{l}\text { CMC-nZVI, } \mathrm{Na}_{2} \mathrm{~S}, \\
\text { two-step }\end{array}$ & Dosed 0.017 & n.a. & 7.1 & $\begin{array}{l}\text { 1,1-DCA, ethane, } \\
\text { ethene }\end{array}$ & $\begin{array}{c}\text { Reduced with decreased CMC } \\
\text { loading and after aging }\end{array}$ & n.a. & $\begin{array}{c}\text { (Nezamabadi et al., } \\
\text { 2016) }\end{array}$ \\
\hline CT & $\begin{array}{l}\text { CMC-nZVI, } \mathrm{Na}_{2} \mathrm{~S}, \\
\text { two-step }\end{array}$ & Dosed 0.017 & n.a. & 1.9 & CF, methane & n.a. & n.a. & $\begin{array}{c}\text { (Nezamabadi et al., } \\
\text { 2016) }\end{array}$ \\
\hline CT & $\begin{array}{l}\mathrm{nZVI}+\mathrm{Na}_{2} \mathrm{~S}_{2} \mathrm{O}_{3} \\
\quad \text { two-step }\end{array}$ & Dosed 0.025 & 0.23 & $\sim 1$ & n.a. & n.a. & $\begin{array}{l}\text { Direct electron transfer; not } \\
\text { affected by sulfidation }\end{array}$ & (Han and Yan, 2016) \\
\hline CT & $\begin{array}{l}\mathrm{mZVI}+\mathrm{S}^{0}, \text { ball } \\
\text { milling }\end{array}$ & Dosed 0.1 & 0.19 & $\begin{array}{l}\text { A bit slower than } \\
\text { mZVI }\end{array}$ & n.a. & n.a. & n.a. & (Gu et al., 2017) \\
\hline CT & $\begin{array}{l}\mathrm{Fe}^{2+}+\mathrm{Na}_{2} \mathrm{~S}_{2} \mathrm{O}_{4} \\
\quad \text { one-step }\end{array}$ & Dosed 0.14 & n.a. & 1.8 & $\mathrm{CF}$ & $\begin{array}{l}\text { Increased with } \mathrm{pH} \text { and } \\
\text { temperature }\end{array}$ & n.a. & (Jin et al., 2018) \\
\hline $\mathrm{CF}$ & $\begin{array}{l}\text { CMC-nZVI, } \mathrm{Na}_{2} \mathrm{~S}, \\
\text { two-step }\end{array}$ & Dosed 0.017 & n.a. & 2.4 & DCM, methane & n.a. & n.a. & $\begin{array}{c}\text { (Nezamabadi et al., } \\
\text { 2016) }\end{array}$ \\
\hline cis-DCE & $\begin{array}{l}\mathrm{Fe}^{2+}+\mathrm{Na}_{2} \mathrm{~S}_{2} \mathrm{O}_{4}, \\
\text { one-step }\end{array}$ & Dosed 0.1 & n.a. & 0.26 & Ethene; ethane; VC & n.a. & $\begin{array}{c}\text { cis-DCE cannot accept electron } \\
\text { at FeS sites }\end{array}$ & $\begin{array}{c}\text { (Mangayayam et al., } \\
\text { 2019a) }\end{array}$ \\
\hline PCE & $\begin{array}{l}\mathrm{mZVI}+\mathrm{Na}_{2} \mathrm{~S}_{2} \mathrm{O}_{3}, \\
\text { two-step }\end{array}$ & Dosed 0.05 & n.a. & 1.4 & Ethene, ethane, $\mathrm{C} 3-\mathrm{C} 6$ & $\begin{array}{l}\text { Increased by Cu-amendment } \\
\text { but highly reduced with } \\
\text { following sulfidation }\end{array}$ & n.a. & (Islam et al., 2020) \\
\hline cis-DCE & $\begin{array}{l}\mathrm{mZVI}+\mathrm{Na}_{2} \mathrm{~S}_{2} \mathrm{O}_{3}, \\
\text { two-step }\end{array}$ & Dosed 0.05 & n.a. & 13.4 & Ethene, ethane, C3-C6 & $\begin{array}{l}\text { Increased by } \mathrm{Cu} \text {-amendment } \\
\text { but highly reduced with } \\
\text { following sulfidation }\end{array}$ & n.a. & \\
\hline
\end{tabular}

* The proposed mechanism for enhanced reactivity of S-(n)ZVI to (n)ZVI. 
Table S4. Summary of the Data on the Degradation of Contaminants (Nonmetal) other than Chlorinated Hydrocarbons (CHCs) by S-(n)ZVI under Anoxic Conditions.

\begin{tabular}{|c|c|c|c|c|c|c|c|c|}
\hline Target & $\begin{array}{c}\text { Sulfidation } \\
\text { method }\end{array}$ & $\begin{array}{l}\text { Optima } \\
\text { I S/Fe }\end{array}$ & $\begin{array}{c}\text { HER, } \\
k_{\mathrm{S}-(\mathrm{n}) \mathrm{ZVI} / \boldsymbol{k}(\mathrm{n}) \mathrm{ZVI},} \\
\mu \mathrm{mol} \cdot \mathrm{L} \cdot \mathrm{g}^{-1} .\end{array}$ & $\begin{array}{c}\text { Contaminant } \\
k_{\mathrm{s}-(\mathrm{n}) \mathrm{ZVI} / \boldsymbol{k}_{(\mathrm{n}) \mathrm{ZVI}},} \\
\mathbf{L} \cdot \mathbf{g}^{-1} \cdot \mathbf{h}^{-1}\end{array}$ & Products & Influencing factors & Proposed mechanisms* & References \\
\hline \multicolumn{9}{|c|}{ S-(n)ZVI, anoxic, contaminants other than CHCs } \\
\hline p-Nitrophenol & $\begin{array}{l}\text { nZVI + } \mathrm{Na}_{2} \mathrm{~S} \\
\text { two-step }\end{array}$ & $\begin{array}{c}\text { Dosed } \\
0.14\end{array}$ & n.a. & 1.46 & $\begin{array}{l}p \text {-Aminophenol, } p- \\
\text { benzoquinone }\end{array}$ & Reduced with increasing $\mathrm{pH}$ & Increased hydrophobicity & (Tang et al., 2016) \\
\hline Indigo disulfonate & $\begin{array}{c}\text { CMC-nZVI, } \\
\mathrm{Na}_{2} \mathrm{~S}_{2} \mathrm{O}_{4} \text { or } \\
\mathrm{Na}_{2} \mathrm{~S} \text {, two-step }\end{array}$ & $\begin{array}{c}\text { Dosed } \\
0.19 \text { or } \\
1\end{array}$ & Negligible & 0.58 & $\begin{array}{c}\text { Dihydro Indigo } \\
\text { disulfonate }\end{array}$ & Reduced after aging & $\begin{array}{l}\text { Sulfidation eliminates reaction } \\
\text { with water }\end{array}$ & (Fan et al., 2016) \\
\hline $\begin{array}{l}\text { Tetrabromobiphen } \\
\text { ol A (TBBPA) }\end{array}$ & $\begin{array}{l}\mathrm{Fe}^{2+}+\mathrm{Na}_{2} \mathrm{~S}_{2} \mathrm{O}_{4}, \\
\text { one-step }\end{array}$ & $\begin{array}{c}\text { Dosed } \\
0.51\end{array}$ & n.a. & 1.65 & $\begin{array}{c}\text { Tri-BBPA, di- } \\
\text { BBPA, mono- } \\
\text { BBPA, and BPA }\end{array}$ & $\begin{array}{l}\text { Reduced to } 56 \% \text { of the initial } \\
\text { transformation after aging for } \\
11 \text { weeks }\end{array}$ & $\begin{array}{l}\text { Increased electrical conductivity, } \\
\text { hydrophobicity, and surface area }\end{array}$ & (Li et al., 2016) \\
\hline $\begin{array}{l}\text { Hexabromocyclod } \\
\text { odecane (HBCD) }\end{array}$ & $\begin{array}{l}\mathrm{Fe}^{2+}+\mathrm{Na}_{2} \mathrm{~S}_{2} \mathrm{O}_{4}, \\
\text { one-step }\end{array}$ & $\begin{array}{c}\text { Dosed } \\
0.2\end{array}$ & n.a. & 1.35 & $\begin{array}{c}\text { Tetrabromo } \\
\text { cyclododecene, } \\
\text { dibromocyclobutane }\end{array}$ & $\begin{array}{l}\text { Reduced with } \mathrm{pH}(5.0,7.0 \text {, and } \\
\text { 9.0), } \mathrm{Ca}^{2+}, \mathrm{Mg}^{2+}, \mathrm{NO}_{3}^{-}, \mathrm{HCO}_{3}^{-}, \\
\mathrm{Cl}^{-} \text {, and IS }\end{array}$ & n.a. & (Li et al., 2017) \\
\hline Florfenicol (FF) & $\begin{array}{c}\mathrm{Fe}^{2+}+\mathrm{Na}_{2} \mathrm{~S}_{2} \mathrm{O}_{4}, \\
\text { one-step }\end{array}$ & $\begin{array}{c}\text { Dosed } \\
0.14\end{array}$ & n.a. & 48 & $\begin{array}{l}\text { Dideschloro FF; } \\
\text { deschloro FF as } \\
\text { intermediate }\end{array}$ & $\begin{array}{l}\text { Optimal at pH 7.0; increased } \\
\text { with temperature; not affected } \\
\text { by dissolved ions and OM }\end{array}$ & $\begin{array}{l}\text { Increased electrical conductivity } \\
\text { and hydrophobicity }\end{array}$ & (Cao et al., 2017) \\
\hline $\begin{array}{c}\text { Organic dyes (azo, } \\
\text { anthraquinone, } \\
\text { triphenylmethane } \\
\text { dyes) }\end{array}$ & $\begin{array}{l}\mathrm{mZVI}+\mathrm{S}^{0}, \text { ball } \\
\text { milling }\end{array}$ & $\begin{array}{c}\text { Dosed } \\
0.05\end{array}$ & n.a. & $1.3-4$ & n.a. & $\begin{array}{c}\text { Reduced with increasing initial } \\
\mathrm{pH}\end{array}$ & n.a. & (Wang et al., 2018) \\
\hline $\begin{array}{c}\mathrm{N}- \\
\text { nitrosodimethylam } \\
\text { ine (NDMA) }\end{array}$ & $\begin{array}{l}\text { mZVI }+\mathrm{Na}_{2} \mathrm{~S}, \\
\text { two-step }\end{array}$ & $\begin{array}{c}\text { Dosed } \\
0.00056\end{array}$ & 0.073 & $\sim 1$ & $\begin{array}{c}\text { Dimethylamine; } \\
\mathrm{N}, \mathrm{N}- \\
\text { dimethylhydrazine } \\
\text { as intermediate }\end{array}$ & $\begin{array}{c}\text { Not affected by presence of } \\
\text { nitrate }\end{array}$ & n.a. & \multirow[t]{2}{*}{ (Qin et al., 2019) } \\
\hline Nitrate $\left(\mathrm{NO}_{3}^{-}\right)$ & $\begin{array}{l}\mathrm{mZVI}+\mathrm{Na}_{2} \mathrm{~S} \\
\text { two-step }\end{array}$ & $\begin{array}{l}\text { Dosed } \\
0.00056\end{array}$ & 0.073 & 0.01 & $\mathrm{NH}_{4}{ }^{+}$ & n.a. & $\begin{array}{l}\text { FeS on the surface reduces nitrate } \\
\text { slowly }\end{array}$ & \\
\hline Nitrate $\left(\mathrm{NO}_{3}^{-}\right)$ & $\begin{array}{l}\mathrm{Fe}^{3+}+\mathrm{Na}_{2} \mathrm{~S}_{2} \mathrm{O}_{4} \\
\text { one-step }\end{array}$ & $\begin{array}{c}\text { Dosed } \\
0.14 ; \\
\text { particle }\end{array}$ & 0.03 & $\begin{array}{l}\text { almost no } \\
\text { reaction by S- } \\
\text { nZVI }\end{array}$ & $\mathrm{NH}_{4}^{+}$ & $\begin{array}{l}\text { Not much affected by aging in } \\
\text { water for } 60 \text { days }\end{array}$ & $\begin{array}{l}\text { Increased hydrophobicity reduces } \\
\text { the reactivity of hydrophilic } \mathrm{NO}_{3}^{-}\end{array}$ & \multirow[t]{2}{*}{ (Xu et al., 2019a) } \\
\hline Nitrate $\left(\mathrm{NO}_{3}^{-}\right)$ & $\begin{array}{l}\text { nZVI }+\mathrm{Na}_{2} \mathrm{~S}, \\
\text { two-step }\end{array}$ & $\begin{array}{c}\text { Dosed } \\
0.14 ; \\
\text { particle } \\
0.008\end{array}$ & 0.17 & 0.32 & $\mathrm{NH}_{4}{ }^{+}$ & $\begin{array}{l}\text { Not much affected by aging in } \\
\text { water for } 60 \text { days }\end{array}$ & $\begin{array}{l}\text { Increased hydrophobicity reduces } \\
\text { the reactivity of hydrophilic } \mathrm{NO}_{3}^{-}\end{array}$ & \\
\hline $\begin{array}{c}2,2^{\prime}, 4,4^{\prime}, 5,5^{\prime}- \\
\text { Hexachlorobiphen } \\
\text { yl (PCB153) }\end{array}$ & $\begin{array}{l}\mathrm{Fe}^{3+}+\mathrm{Na}_{2} \mathrm{~S}_{2} \mathrm{O}_{4} \\
\text { one-step }\end{array}$ & $\begin{array}{c}\text { Dosed } \\
0.1\end{array}$ & n.a. & 0.83 & Tetrachlorobiphenyls & Not affected by $\mathrm{Cu}^{2+}$ and $\mathrm{Ni}^{2+}$ & n.a. & (Lou et al., 2019) \\
\hline $\begin{array}{l}\text { Decabromodiphen } \\
\text { yl ether (BDE- } \\
\text { 209) }\end{array}$ & $\begin{array}{c}\mathrm{Fe}^{3+}+\mathrm{Na}_{2} \mathrm{~S}_{2} \mathrm{O}_{4} \\
\text { one-step }\end{array}$ & $\begin{array}{c}\text { Dosed } \\
0.2\end{array}$ & n.a. & 7.1 & $\begin{array}{l}\text { Less-brominated } \\
\text { products }\end{array}$ & $\begin{array}{l}\text { Reduced by DO and increased } \\
\text { with increasing initial } \mathrm{pH} \text { and } \\
\text { presence of tetrahydrofuran }\end{array}$ & $\begin{array}{l}\text { Reduced HER and increased } \\
\text { electrical conductivity to target } \\
\text { contaminants }\end{array}$ & (Wei et al., 2019) \\
\hline
\end{tabular}




\begin{tabular}{|c|c|c|c|c|c|c|c|c|}
\hline Nitrobenzene & $\begin{array}{l}\mathrm{Fe}^{2+}+\mathrm{Na}_{2} \mathrm{~S}_{2} \mathrm{O}_{4} \\
\text { one-step }\end{array}$ & $\begin{array}{c}\text { Dosed } \\
0.3\end{array}$ & n.a. & 7.4 & Aniline & n.a. & $\begin{array}{c}\text { Reduced biotoxicity and } \\
\text { increased electrical conductivity }\end{array}$ & (Zhang et al., 2020) \\
\hline $\begin{array}{l}\text { Chloramphenicol } \\
\text { (CAP) }\end{array}$ & $\begin{array}{l}\text { nZVI + Na2S, } \\
\text { two-step }\end{array}$ & $\begin{array}{c}\text { Dosed } \\
0.14 ; \\
\text { particle } \\
0.0028 \\
\end{array}$ & 0.06 & $\begin{array}{l}\text { Substantially } \\
\text { reduced }\end{array}$ & Denitration product & n.a. & Increased hydrophobicity & \multirow[t]{4}{*}{ (Cao et al., 2020a) } \\
\hline Florfenicol (FF) & $\begin{array}{l}\text { nZVI + } \mathrm{Na}_{2} \mathrm{~S}, \\
\text { two-step }\end{array}$ & $\begin{array}{c}\text { Dosed } \\
0.14 ; \\
\text { particle } \\
0.0028 \\
\end{array}$ & 0.06 & 4 & $\begin{array}{l}\text { Dechlorination } \\
\text { product }\end{array}$ & Reduced by aging & Increased hydrophobicity & \\
\hline $\begin{array}{c}\text { Chloramphenicol } \\
\text { (CAP) }\end{array}$ & $\begin{array}{l}\mathrm{Fe}^{2+}+\mathrm{Na}_{2} \mathrm{~S}_{2} \mathrm{O}_{4} \\
\text { one-step }\end{array}$ & $\begin{array}{c}\text { Dosed } \\
0.28\end{array}$ & n.a. & 0.033 & Denitration product & $\begin{array}{l}\text { Decreased with increasing } \\
\text { hydrophobicity }\end{array}$ & Increased hydrophobicity & \\
\hline Florfenicol (FF) & $\begin{array}{l}\mathrm{Fe}^{2+}+\mathrm{Na}_{2} \mathrm{~S}_{2} \mathrm{O}_{4} \\
\text { one-step }\end{array}$ & $\begin{array}{c}\text { Dosed } \\
0.14 ; \\
\text { particle } \\
0.035 \\
\end{array}$ & $\begin{array}{ll}\text { n.a. } \\
\text {. }\end{array}$ & 8 & $\begin{array}{l}\text { Dechlorination } \\
\text { product }\end{array}$ & $\begin{array}{l}\text { Increased with increasing } \\
\text { hydrophobicity }\end{array}$ & Increased hydrophobicity & \\
\hline Diatrizoate (DTA) & $\begin{array}{l}\mathrm{Fe}^{3+}+\mathrm{Na}_{2} \mathrm{~S}_{2} \mathrm{O}_{4} \\
\text { one-step }\end{array}$ & $\begin{array}{c}\text { Dosed } \\
0.25\end{array}$ & $\begin{array}{c}0.12\left(\mathrm{H}_{2}\right. \\
\text { generation } \\
\text { within } 48 \mathrm{~h})\end{array}$ & 5.2 & $\begin{array}{c}\text { Deiodination } \\
\text { product: } 3.5- \\
\text { bis(acetylamino)ben } \\
\text { zoic acid }\end{array}$ & Reduced by HA and in GW & $\begin{array}{l}\text { Poisoning of } \mathrm{H}^{*} \text { recombination } \\
\text { by sulfur; increased electron } \\
\text { conductivity }\end{array}$ & (Zhou et al., 2020) \\
\hline Florfenicol (FF) & $\begin{array}{l}\mathrm{Fe}^{3+}+\mathrm{Na}_{2} \mathrm{~S}_{2} \mathrm{O}_{4} \\
\text { one-step }\end{array}$ & $\begin{array}{c}\text { Dosed } \\
0.21 ; \\
\text { particle } \\
0.095\end{array}$ & Highly reduced & 12.5 & $\begin{array}{l}\text { Dechlorinated and } \\
\text { defluorinated } \\
\text { product }\end{array}$ & n.a. & Increased hydrophobicity & \multirow[t]{3}{*}{ (Cao et al., 2020b) } \\
\hline Florfenicol (FF) & $\begin{array}{c}\mathrm{nZVI}+ \\
\mathrm{Na}_{2} \mathrm{~S}_{2} \mathrm{O}_{4}, \text { two- } \\
\text { step }\end{array}$ & $\begin{array}{c}\text { Dosed } \\
0.07 \\
\text { particle } \\
0.003\end{array}$ & 0.21 & 9.3 & $\begin{array}{l}\text { Dechlorinated and } \\
\text { defluorinated } \\
\text { product }\end{array}$ & n.a. & Increased hydrophobicity & \\
\hline Florfenicol (FF) & $\begin{array}{l}\text { nZVI }+\mathrm{Na}_{2} \mathrm{~S}, \\
\text { two-step }\end{array}$ & $\begin{array}{c}\text { Dosed } \\
0.07 \\
\text { particle } \\
0.008 \\
\end{array}$ & 0.15 & 11.3 & $\begin{array}{l}\text { Dechlorinated and } \\
\text { defluorinated } \\
\text { product }\end{array}$ & n.a. & Increased hydrophobicity & \\
\hline p-nitrophenol & $\begin{array}{l}\text { nZVI }+\mathrm{Na}_{2} \mathrm{~S}, \\
\text { two-step }\end{array}$ & $\begin{array}{c}\text { Dosed } \\
0.1\end{array}$ & n.a. & 16 & $\begin{array}{c}\text { Reduction into } p- \\
\text { aminophenol (90\%); } \\
\text { oxidation to } p- \\
\text { benzoquinone (10\%) } \\
\end{array}$ & n.a. & $\begin{array}{l}\mathrm{H}_{2} \mathrm{O}_{2} \text { is formed from reaction of } \\
\text { water and } \mathrm{Fe} S_{2} \text { and then } \\
\text { generates } \\
\text { by through activation } \\
\text { by surface-bound } \mathrm{Fe}(\mathrm{II})\end{array}$ & (Tian et al., 2021) \\
\hline \multicolumn{9}{|l|}{ Support } \\
\hline Nitrobenzene & $\begin{array}{l}\mathrm{Biochar} \mathrm{Fe}^{2+}+ \\
\mathrm{Na}_{2} \mathrm{~S}_{2} \mathrm{O}_{4} \text {, one- } \\
\text { step }\end{array}$ & $\begin{array}{c}\text { Dosed } \\
0.3\end{array}$ & n.a. & n.a. & Aniline & $\begin{array}{l}\text { Increased by biochar support; } \\
\text { reduced to } 72.2 \% \text { of the initial } \\
\text { removal by aging in air for } 12 \\
\text { weeks }\end{array}$ & n.a. & (Zhang et al., 2018) \\
\hline Methyl orange & $\begin{array}{l}\text { Biochar, nZVI } \\
+\mathrm{Na}_{2} \mathrm{~S}_{2} \mathrm{O}_{4} \text {, two- } \\
\text { step }\end{array}$ & $\begin{array}{c}\text { Dosed } \\
0.5\end{array}$ & n.a. & 1.44 & n.a. & $\begin{array}{c}\text { Reduced by } \mathrm{Cl}^{-}, \mathrm{Ca}^{2+}, \mathrm{NH}_{4}^{+} \text {, } \\
\text { HA, and DO }\end{array}$ & n.a. & (Yang et al., 2018) \\
\hline Nitrobenzene & $\begin{array}{c}\text { Modified } \\
\text { biochar, } \\
\mathrm{Na}_{2} \mathrm{~S}_{2} \mathrm{O}_{4} \text {, one- } \\
\text { step }\end{array}$ & $\begin{array}{c}\text { Dosed } \\
0.3\end{array}$ & n.a. & n.a. & n.a. & $\begin{array}{l}\text { Increased by pretreatment of } \\
\text { biochar with } \mathrm{HCl} \text { and } \mathrm{NaOH}\end{array}$ & n.a. & (Zhang et al., 2019) \\
\hline
\end{tabular}




\begin{tabular}{|c|c|c|c|c|c|c|c|c|}
\hline Florfenicol (FF) & $\begin{array}{c}\mathrm{Fe}^{2+}+\mathrm{Na}_{2} \mathrm{~S}_{2} \mathrm{O}_{4} \\
\text { one-step } \\
\text { biochar }\end{array}$ & $\begin{array}{c}\text { Dosed } \\
0.14\end{array}$ & n.a. & $\begin{array}{c}\text { Highly } \\
\text { improved; no } \\
\text { degradation by } \\
\text { nZVI }\end{array}$ & $\begin{array}{c}\text { Dideschloro FF and } \\
\text { deschloro FF as } \\
\text { intermediate }\end{array}$ & $\begin{array}{l}\text { Increased by biochar support; } \\
\text { DO stops the reaction of FF }\end{array}$ & $\begin{array}{l}\text { Increased electrical conductivity } \\
\text { and hydrophobicity }\end{array}$ & (Xu et al., 2019d) \\
\hline Bromate $\left(\mathrm{BrO}_{3}^{-}\right)$ & $\begin{array}{c}\text { Algal carbon, } \\
\mathrm{Fe}(\mathrm{II}), \mathrm{Na}_{2} \mathrm{~S}_{2} \mathrm{O}_{4}, \\
\text { high } \\
\text { temperature }\end{array}$ & $\begin{array}{c}\text { Dosed } \\
0.3\end{array}$ & n.a. & 3 & Bromide & $\begin{array}{c}\text { Increased by algal carbon } \\
\text { support, temperature, and ionic } \\
\text { strength; reduced by increasing } \\
\text { pH }\end{array}$ & n.a. & (Lu et al., 2020) \\
\hline Florfenicol (FF) & $\begin{array}{c}\mathrm{nZVI}+\mathrm{Fe}^{2+} \\
\mathrm{Na}_{2} \mathrm{~S}, \text { two-step }\end{array}$ & $\begin{array}{c}\text { Dosed } \\
0.07 ; \\
\text { particle } \\
0.05\end{array}$ & 0.02 & $\begin{array}{c}3 \\
\text { (defluorination } \\
\text { extent) }\end{array}$ & $\begin{array}{c}\text { Dideschloro FF and } \\
\text { deschloro FF as } \\
\text { intermediate; } \\
\text { defluorination } \\
\text { product }\end{array}$ & n.a. & $\begin{array}{l}\text { S induces chemically reactive } \\
\text { surface and changes the } \\
\text { dechlorination pathway from } \\
\text { atomic } \mathrm{H} \text { to electron transfer }\end{array}$ & (Cao et al., 2021) \\
\hline
\end{tabular}

* The proposed mechanism for enhanced reactivity of S-(n)ZVI to (n)ZVI.

n.a. not available; HA, humic acid; GW, groundwater; IS, ionic strength; OM, organic matter; DO, dissolved oxygen; HER, hydrogen evolution rate.

Table S5. Summary of the Data on the Degradation of Contaminants by S-(n)ZVI under Oxic Conditions.

\begin{tabular}{|c|c|c|c|c|c|c|c|c|c|}
\hline Target & $\begin{array}{l}\text { Sulfidation } \\
\text { method }\end{array}$ & $\begin{array}{c}\text { Optimal } \\
\text { S/Fe }\end{array}$ & Oxidant & $\begin{array}{l}\text { Responsible } \\
\text { process }\end{array}$ & $\begin{array}{l}\text { Contaminant } \\
k_{\mathrm{S}-(\mathrm{n}) \mathrm{ZV}} \mathbf{k} k_{(\mathrm{n}) \mathrm{ZVI}}, \\
\mathbf{L} \cdot \mathbf{g}^{-1} \cdot \mathbf{h}^{-1}\end{array}$ & Products & Influencing factors & Proposed mechanisms* & References \\
\hline \multicolumn{10}{|c|}{ S-(n)ZVI, oxic, Fe ${ }^{0}$ excess } \\
\hline $\begin{array}{c}\text { Bisphenol } \\
\text { A }\end{array}$ & $\begin{array}{l}\mathrm{Na}_{2} \mathrm{~S}_{2} \mathrm{O}_{3}+ \\
\text { oxalate }\end{array}$ & $\begin{array}{c}\text { Dosed } \\
1\end{array}$ & $\mathrm{H}_{2} \mathrm{O}_{2}$ & $\begin{array}{c}\text { Oxidation by } \\
\text { OH }\end{array}$ & 25 & $\begin{array}{c}\text { Complete } \\
\text { mineralization }\end{array}$ & n.a. & n.a. & (Du et al., 2016) \\
\hline $\begin{array}{l}\mathrm{p}- \\
\text { Nitrophen } \\
\text { ol }\end{array}$ & $\begin{array}{l}\text { nZVI + } \mathrm{Na}_{2} \mathrm{~S}, \\
\text { two-step }\end{array}$ & $\begin{array}{l}\text { Dosed } \\
0.14\end{array}$ & $\mathrm{O}_{2}$ & $\begin{array}{l}\text { Reduction and } \\
\text { oxidation by } \\
\text { 'OH }\end{array}$ & 3.2 & $\begin{array}{c}p \text {-Aminophenol, } p \text { - } \\
\text { benzoquinone, and } \\
\text { hydroquinone, and } \\
\text { acetic acid } \\
\end{array}$ & Reduced with increasing $\mathrm{pH}$ & Increased hydrophobicity & (Tang et al., 2016) \\
\hline Orange I & $\begin{array}{l}\mathrm{mZVI+} \\
\mathrm{Na}_{2} \mathrm{~S}, \text { two- } \\
\text { step }\end{array}$ & $\begin{array}{c}\text { Dosed } \\
0.083\end{array}$ & $\mathrm{O}_{2}$ & Reduction & 22.5 & $\begin{array}{l}\text { Sulfanilic acid, 1- } \\
\text { amino-4-naphthol }\end{array}$ & $\begin{array}{l}\text { Increased by application of a } \\
\text { weak magnetic field }\end{array}$ & n.a. & (Xu et al., 2016) \\
\hline $\begin{array}{l}\text { Rhodamin } \\
\text { e B }\end{array}$ & $\begin{array}{c}\text { nZVI + } \\
\text { thioacetamid } \\
\text { e, two-step }\end{array}$ & $\begin{array}{c}\text { Dosed } \\
0.24\end{array}$ & $\mathrm{O}_{2}$ & $\begin{array}{l}\text { Reduction and } \\
\text { oxidation by } \\
\text { 'OH }\end{array}$ & 1.8 & n.a. & Optimal at $\mathrm{pH} 4.65-6.68$ & n.a. & $\begin{array}{l}\text { (Zhang et al., } \\
\text { 2016) }\end{array}$ \\
\hline $\begin{array}{l}\text { Diclofenac } \\
\text { (DCF) }\end{array}$ & $\begin{array}{l}\mathrm{Fe}^{3+}+ \\
\mathrm{Na}_{2} \mathrm{~S}_{2} \mathrm{O}_{4} \\
\text { one-step }\end{array}$ & $\begin{array}{c}\text { Dosed } \\
0.3\end{array}$ & $\mathrm{O}_{2}$ & $\begin{array}{l}\text { Adsorption and } \\
\text { oxidation by } \\
\text { 'OH }\end{array}$ & 3.5 & n.a. & $\begin{array}{c}\text { Reduced with increasing } \mathrm{pH} \text { and } \\
\mathrm{HA}, \mathrm{NO}_{3}^{-}, \mathrm{SO}_{4}^{2-}, \mathrm{HPO}_{4}^{2^{--}} \text {, and } \\
\mathrm{HCO}_{3}^{--}\end{array}$ & $\begin{array}{c}\text { Reduced reaction with } \mathrm{O}_{2} \text { and } \\
\text { increased electrical conductivity to } \\
\text { surface } \mathrm{Fe}(\mathrm{III})\end{array}$ & (Song et al., 2017) \\
\hline $\begin{array}{c}\text { Benzoic } \\
\text { acid }\end{array}$ & $\begin{array}{l}\mathrm{Fe}^{3+}+ \\
\mathrm{Na}_{2} \mathrm{~S}_{2} \mathrm{O}_{4} \\
\text { one-step }\end{array}$ & $\begin{array}{c}\text { Dosed } \\
0.08\end{array}$ & PDS & $\begin{array}{l}\text { Oxidation by } \\
\mathrm{O}^{\mathrm{OH}} \text { (dominant) } \\
\text { and } \mathrm{SO}_{4}^{-}\end{array}$ & Slight increase & $\begin{array}{c}\text { Complete } \\
\text { mineralization }\end{array}$ & $\begin{array}{l}\text { Reduced with increasing } \mathrm{pH}, \text { and } \\
\text { by organic ligands, } \mathrm{SO}_{4}^{2-} \\
\mathrm{HCO}_{3}^{-}, \mathrm{Cl}^{-} \text {and } \mathrm{NO}_{3}^{-}\end{array}$ & Increased iron dissolution & $\begin{array}{l}\text { (Rayaroth et al., } \\
\text { 2017) }\end{array}$ \\
\hline $\begin{array}{l}\text { Diclofenac } \\
\text { (DCF) }\end{array}$ & $\begin{array}{l}\mathrm{Fe}^{3+}+ \\
\mathrm{Na}_{2} \mathrm{~S}_{2} \mathrm{O}_{4} \\
\text { one-step }\end{array}$ & $\begin{array}{c}\text { Dosed } \\
0.3\end{array}$ & $\mathrm{O}_{2}$ & $\begin{array}{l}\text { Adsorption and } \\
\text { oxidation }\end{array}$ & 3.5 & n.a. & n.a. & $\begin{array}{l}\text { Reduced reaction with } \mathrm{O}_{2} \text { and } \\
\text { increased electron transfer to } \\
\text { surface } \mathrm{Fe}(\mathrm{IIII}) \text { for generation of } \\
\mathrm{H}_{2} \mathrm{O}_{2} \text { and } \mathrm{OH}\end{array}$ & (Su et al., 2018) \\
\hline $\begin{array}{l}\text { Acid red } \\
73\end{array}$ & $\begin{array}{l}\mathrm{mZVI}+ \\
\mathrm{Na}_{2} \mathrm{~S} \text {, two- } \\
\text { step }\end{array}$ & $\begin{array}{l}\text { Dosed } \\
0.084\end{array}$ & $\mathrm{O}_{2}$ & Reduction & 2.3 & $\begin{array}{l}\text { 4-Amino azobenzene, } \\
\text { p-phenylenediamine, } \\
\text { aniline }\end{array}$ & $\begin{array}{c}\text { Increased by coupling with } \\
\text { EDTA }\end{array}$ & Increased electrical conductivity & $\begin{array}{l}\text { (Zhang et al., } \\
\text { 2019) }\end{array}$ \\
\hline
\end{tabular}




\begin{tabular}{|c|c|c|c|c|c|c|c|c|c|}
\hline $\begin{array}{c}\text { Tetrabrom } \\
\text { obisphenol } \\
\text { A }\end{array}$ & $\begin{array}{l}\text { nZVI + } \mathrm{Na}_{2} \mathrm{~S} \\
\text { two-step }\end{array}$ & $\begin{array}{c}\text { Dosed } \\
0.3\end{array}$ & $\begin{array}{l}\text { Anoxic } \\
+ \text { oxic } \\
\left(\mathrm{O}_{2}\right)\end{array}$ & $\begin{array}{l}\text { Reduction and } \\
\text { then oxidation } \\
\text { by }{ }^{\circ} \mathrm{OH}\end{array}$ & 1.5 & $\begin{array}{l}\text { Biphenol A (anoxic); } \\
\text { dihydroxybenzenes and } \\
\text { benzoquinones (oxic) }\end{array}$ & Reduced with increasing $\mathrm{pH}$ & $\begin{array}{l}\text { Reduced HER (anoxic) and } \\
\text { increased }{ }^{\circ} \mathrm{OH} \text { production by } \\
\text { surface-bound } \mathrm{Fe}(\mathrm{II}) \text { (oxic) }\end{array}$ & (Wu et al., 2019) \\
\hline TCE & $\begin{array}{c}\mathrm{Fe}^{3+}+ \\
\mathrm{Na}_{2} \mathrm{~S}_{2} \mathrm{O}_{4} \\
\text { one-step }\end{array}$ & $\begin{array}{c}\text { Dosed } \\
0.033-0.0 \\
4\end{array}$ & PDS & $\begin{array}{c}\text { Oxidation by } \\
\mathrm{OH}^{\circ} \text { and } \mathrm{SO}^{-}{ }^{-} \\
\text {(acidic } \mathrm{pH})\end{array}$ & 1.2 & Formic acid & Reduced in GW & Increased iron dissolution rates & $\begin{array}{l}\text { (Dong et al., } \\
\text { 2019a) }\end{array}$ \\
\hline $\begin{array}{l}\text { Sulfameth } \\
\text { azine }\end{array}$ & $\begin{array}{l}\mathrm{Fe}^{3+}+ \\
\mathrm{Na}_{2} \mathrm{~S}_{2} \mathrm{O}_{4} \\
\text { one-step }\end{array}$ & $\begin{array}{c}0.017 \\
(\mathrm{PDS}) \\
0.01-0.1 \\
\left(\mathrm{H}_{2} \mathrm{O}_{2}\right)\end{array}$ & $\begin{array}{l}\mathrm{PDS} ; \\
\mathrm{H}_{2} \mathrm{O}_{2}\end{array}$ & Oxidation & n.a. & n.a. & $\begin{array}{l}\text { Reactivity of S-nZVI/PDS is not } \\
\text { affected by initial pH; } \mathrm{S}- \\
\text { nZVI/ } / \mathrm{H}_{2} \mathrm{O}_{2} \text { only works at } \mathrm{pH} 3\end{array}$ & n.a. & $\begin{array}{l}\text { (Dong et al., } \\
2019 \mathrm{~b} \text { ) }\end{array}$ \\
\hline $\begin{array}{c}\text { Sulfameth } \\
\text { oxazole }\end{array}$ & $\begin{array}{l}\mathrm{mZVI}+ \\
\mathrm{Na}_{2} \mathrm{~S}, \text { two- } \\
\text { step }\end{array}$ & $\begin{array}{c}\text { Dosed } \\
0.1\end{array}$ & PMS & $\begin{array}{c}\text { Oxidation by } \\
\mathrm{SO}_{4}^{{ }^{-}} \\
\text {(dominant) and } \\
{ }^{\circ} \mathrm{OH}\end{array}$ & 2.7 & $\begin{array}{l}\text { Multiple intermediates } \\
\text { and products }\end{array}$ & $\begin{array}{l}\text { Reduced with increasing } \mathrm{pH} \text { and } \\
\text { by } \mathrm{SO}_{4}{ }^{2-}, \mathrm{HCO}_{3}^{-}, \mathrm{Cl}^{-} \text {and } \mathrm{NO}_{3}^{-} \\
\text {increased by DO }\end{array}$ & Increased electrical conductivity & (Li et al., 2019) \\
\hline Sulfameth & $\begin{array}{l}\text { mZVI }+\mathrm{S}^{0} \text {, } \\
\text { ball milling }\end{array}$ & $\begin{array}{c}\text { Dosed } \\
0.05\end{array}$ & PDS & $\begin{array}{c}\text { Oxidation by } \\
\mathrm{SO}_{4}^{{ }^{-}} \\
\text {(dominant) and } \\
{ }^{\circ} \mathrm{OH}\end{array}$ & 3.8 & $\begin{array}{l}\text { Oxidation products of } \\
\mathrm{S}-\mathrm{N} \text { bond and }-\mathrm{NH}_{2} \\
\text { group }\end{array}$ & $\begin{array}{l}\text { Reduced with increasing } \mathrm{pH} ; \\
\text { increased by } \mathrm{SO}_{4}{ }^{2-} \text { and } \mathrm{Cl}^{-} ;\end{array}$ & $\begin{array}{l}\text { Increased conductivity and } \\
\text { oxidability resulting in more } \\
\text { dissolution and circulation of iron }\end{array}$ & (Guo et al., 2020) \\
\hline $\begin{array}{l}\text { Carbamaze } \\
\text { pine }\end{array}$ & $\begin{array}{l}\mathrm{Fe}^{3+}+ \\
\mathrm{Na}_{2} \mathrm{~S}_{2} \mathrm{O}_{4} \\
\text { one-step }\end{array}$ & $\begin{array}{c}\text { Dosed } \\
0.1\end{array}$ & $\begin{array}{l}\mathrm{O}_{2} \text { (with } \\
\text { ligand) }\end{array}$ & $\begin{array}{l}\text { Oxidation by } \\
{ }^{1} \mathrm{O}_{2}\end{array}$ & 2.7 & $\begin{array}{l}\text { Mono- or di- } \\
\text { hydroxylated products, } \\
\text { formic acid, and acetic } \\
\text { acid } \\
\end{array}$ & Optimal pH 8.5; reduced in GW & $\begin{array}{l}\text { FeS serves as the source of } \mathrm{Fe}^{2+} \text { and } \\
\text { links } \mathrm{Fe}^{0} \text { to } \mathrm{Fe}^{3+} \text { for regeneration of } \\
\qquad \mathrm{Fe}^{2+}\end{array}$ & $\begin{array}{l}\text { (Rayaroth et al., } \\
\text { 2020) }\end{array}$ \\
\hline $\begin{array}{l}\text { Acetamino } \\
\text { phen } \\
\text { (ACT) }\end{array}$ & $\begin{array}{l}\mathrm{mZVI}+\mathrm{S}^{0}, \\
\text { ball milling }\end{array}$ & $\begin{array}{c}\text { Dosed } \\
0.05\end{array}$ & PDS & $\begin{array}{l}\text { Oxidation by } \\
\mathrm{SO}_{4}^{-{ }^{-}}, \cdot \mathrm{OH} \text {, and } \\
\mathrm{Fe}(\mathrm{IV})\end{array}$ & 5.1 & n.a. & Increased in the absence of $\mathrm{O}_{2}$ & n.a. & (Qiao et al., 2020) \\
\hline $\begin{array}{l}\text { Sulfameth } \\
\text { azine }\end{array}$ & $\begin{array}{l}\mathrm{Fe}^{3+}+ \\
\mathrm{Na}_{2} \mathrm{~S}_{2} \mathrm{O}_{4} \\
\text { one-step }\end{array}$ & Dosed & $\begin{array}{l}\mathrm{O}_{2} \text { and } \\
\text { sulfite }\end{array}$ & $\begin{array}{l}\text { Oxidation by } \\
\mathrm{O}^{\mathrm{OH}} \text { (dominant) } \\
\text { and } \mathrm{SO}_{4}^{--}\end{array}$ & 1.1 & $\begin{array}{l}\text { Transformed without } \\
\text { being mineralized }\end{array}$ & $\begin{array}{l}\text { DO is essential; increased with } \\
\text { sulfite from } 0 \text { to } 1.0 \mathrm{mM} \text {; reduced } \\
\text { with increasing initial pH; } \\
\text { reduced in } \mathrm{GW} \text { and by } \mathrm{HA} \text {; not } \\
\text { affected by aging }\end{array}$ & n.a. & (Li et al., 2020) \\
\hline
\end{tabular}

n.a. not available; GW, groundwater; OM, organic matter; DO, dissolved oxygen; HA, humic acid; HER, hydrogen evolution rate; EDTA, ethylenediaminetetraacetic acid.

Table S6. Summary of the Data on the Degradation of Metals, Metalloids, and Oxyanions by S-(n)ZVI.

\begin{tabular}{|c|c|c|c|c|c|c|c|c|c|}
\hline Target & $\begin{array}{c}\text { Sulfidation } \\
\text { method }\end{array}$ & $\begin{array}{l}\text { Optima } \\
\text { I S/Fe }\end{array}$ & $\begin{array}{l}\text { Anoxic/oxic } \\
\text { conditions }\end{array}$ & $\begin{array}{c}\text { Responsible } \\
\text { process }\end{array}$ & 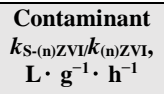 & Products & Influencing factors & Proposed mechanisms* & References \\
\hline $\begin{array}{l}\text { Pertechnetate } \\
\text { (Tc (VII)) }\end{array}$ & $\begin{array}{l}\text { nZVI +Na2S, } \\
\text { two-step }\end{array}$ & $\begin{array}{l}0.056-0 \\
.2\end{array}$ & Anoxic & Reduction & 50 & $\mathrm{TcO}_{2} \cdot \mathrm{nH}_{2} \mathrm{O} ; \mathrm{TcS}_{2}$ & -- & Higher affinity for $\mathrm{FeS}$ & (Fan et al., 2013) \\
\hline $\begin{array}{c}\text { Chromate } \\
\left(\mathrm{Cd}^{2+}\right)\end{array}$ & $\begin{array}{l}\mathrm{Fe}^{3+}+\mathrm{NaS}_{2} \mathrm{O}_{4} \\
\text { one-step }\end{array}$ & 0.28 & Anoxic & Adsorption & 2 & $\mathrm{CdS} ; \mathrm{Fe}_{(1-\mathrm{x})} \mathrm{Cd}_{\mathrm{x}} \mathrm{S}$ & $\begin{array}{l}\text { Not affected by aging; increased with } \mathrm{pH} \\
\text { and by DO }\end{array}$ & -- & (Su et al., 2015) \\
\hline $\begin{array}{c}\text { Chromate } \\
\left(\mathrm{Cd}^{2+}\right)\end{array}$ & $\begin{array}{l}\mathrm{Fe}^{3+}+\mathrm{NaS}_{2} \mathrm{O}_{4}, \\
\quad \text { one-step }\end{array}$ & 0.28 & Anoxic & Adsorption & 2 & -- & $\begin{array}{l}\text { Increased by seeding with nano- } \mathrm{SiO}_{2} \text {, nano- } \\
\qquad \mathrm{TiO}_{2} \text {, and nano- } \mathrm{Al}_{2} \mathrm{O}_{3}\end{array}$ & -- & (Su et al., 2016) \\
\hline $\begin{array}{c}\text { Chromate (Cr } \\
\text { (VI)) }\end{array}$ & $\begin{array}{l}\text { nZVI }+\mathrm{Na}_{2} \mathrm{~S}, \\
\text { two-step }\end{array}$ & 0.056 & Anoxic & Reduction & 14.0 & $\begin{array}{c}\mathrm{Cr}(\mathrm{III}) ; \mathrm{Fe}-\mathrm{Cr} \\
\text { hydroxide precipitates }\end{array}$ & Reduced with increasing $\mathrm{pH}$ and by $\mathrm{DO}$ & $\begin{array}{c}\text { Increased electrical } \\
\text { conductivity }\end{array}$ & (Du et al., 2016) \\
\hline
\end{tabular}




\begin{tabular}{|c|c|c|c|c|c|c|c|c|c|}
\hline $\begin{array}{l}\text { Chromate (Cr } \\
\text { (VI)) }\end{array}$ & $\begin{array}{l}\mathrm{Fe}^{3+}+\mathrm{Na}_{2} \mathrm{~S}_{2} \mathrm{O}_{4} \\
\quad \text { one-step }\end{array}$ & 0.21 & Anoxic & $\begin{array}{l}\text { Adsorption, } \\
\text { reduction, and } \\
\text { immobilization }\end{array}$ & 1.6 & $\begin{array}{c}\mathrm{Cr}(\mathrm{III}) \\
\left(\mathrm{Cr}_{\mathrm{x}} \mathrm{Fe}_{1-\mathrm{x}}\right)(\mathrm{OH})_{3} \\
\mathrm{FeCr}_{2} \mathrm{~S}_{4}\end{array}$ & Reduced with increasing $\mathrm{pH}$ & $\begin{array}{l}\text { Increased surface area; } \\
\text { inhibited aggregation }\end{array}$ & $\begin{array}{l}\text { (Gong et al., } \\
\text { 2017) }\end{array}$ \\
\hline $\begin{array}{c}\text { Chromate (Cr } \\
\text { (VI)) }\end{array}$ & $\begin{array}{l}\text { Biochar + nZVI } \\
+\mathrm{Na}_{2} \mathrm{~S}_{2} \mathrm{O}_{4} \text {, two- } \\
\text { step }\end{array}$ & 0.25 & Anoxic & Adsorption & 3.6 & -- & $\begin{array}{l}\text { Reduced with increasing pH; increased by } \\
\mathrm{SO}_{4}{ }^{2-} ; \text { slightly inhibited by } \mathrm{Ca}^{2+} \text { and } \mathrm{DO} ; \\
\text { not much affected by biochar }\end{array}$ & -- & (Gao et al., 2018) \\
\hline $\begin{array}{l}\text { Antimonite } \\
(\mathrm{Sb}(\mathrm{III}))\end{array}$ & $\begin{array}{l}\mathrm{mZVI}+\mathrm{Na}_{2} \mathrm{~S} \\
\text { two-step }\end{array}$ & 0.084 & Oxic & $\begin{array}{l}\text { Adsorption and } \\
\text { oxidation }\end{array}$ & 15 & $\mathrm{Sb}(\mathrm{V})$ & Not affected by $\mathrm{pH}, \mathrm{Fe}^{2+}$, and aging & $\begin{array}{l}\text { Increased electrical } \\
\text { conductivity }\end{array}$ & $\begin{array}{l}\text { (Huang et al., } \\
\text { 2018) }\end{array}$ \\
\hline $\begin{array}{l}\text { Chromate }(\mathrm{Cr} \\
(\mathrm{VI}))\end{array}$ & $\begin{array}{c}\mathrm{mZVI}+\mathrm{S}^{0} \text {, ball } \\
\text { milling; mZVI } \\
+\mathrm{Na}_{2} \mathrm{~S} \text {, two- } \\
\text { step }\end{array}$ & 0.05 & Oxic & Reduction & $13.3 ; 12.9$ & $\mathrm{Cr}$ (III) & -- & $\begin{array}{c}\text { Increased anion } \\
\text { selectivity and electrical } \\
\text { conductivity }\end{array}$ & (Li et al., 2018) \\
\hline $\begin{array}{c}\text { Chromate } \\
\left(\mathrm{Cd}^{2+}\right)\end{array}$ & $\begin{array}{l}\mathrm{Fe}^{3+}+\mathrm{NaS}_{2} \mathrm{O}_{4} \\
\quad \text { one-step }\end{array}$ & 0.3 & Anoxic & Adsorption & 3.1 & CaS; FeS-Cd & Not affected by $\mathrm{pH}, \mathrm{IS}$, and $\mathrm{HA}$ & $\begin{array}{l}\text { Increased surface area and } \\
\text { reduced aggregation }\end{array}$ & (Lv et al., 2018) \\
\hline $\begin{array}{l}\text { Chromate (Cr } \\
\text { (VI)) }\end{array}$ & $\begin{array}{c}\mathrm{mZVI}+\mathrm{Na}_{2} \mathrm{~S} \\
\text { two-step }\end{array}$ & 0.056 & $\begin{array}{l}\text { Anoxic and } \\
\text { oxic }\end{array}$ & Reduction & $\sim 2$ & $\mathrm{Cr}$ (III) & Increased by the presence of $\mathrm{O}_{2}$ & $\begin{array}{c}\text { Increased electrical } \\
\text { conductivity and release } \\
\text { of } \mathrm{Fe}(\mathrm{II})\end{array}$ & (Shao et al., 2018) \\
\hline $\begin{array}{c}\text { Chromate (Cr } \\
\text { (VI)); } \\
\text { antimony (Sb } \\
\text { (III)) }\end{array}$ & $\begin{array}{c}\mathrm{mZVI}+\mathrm{Na}_{2} \mathrm{~S} \\
\text { two-step }\end{array}$ & 0.056 & Oxic & $\begin{array}{c}\text { Reduction, } \\
\text { oxidation, } \\
\text { adsorption, and } \\
\text { coprecipitation }\end{array}$ & $90 ; 7$ & $\mathrm{Cr}$ (III); Sb (V) & Optimal at pH 6; increased by Fe (II) & -- & $\begin{array}{l}\text { (Wang et al., } \\
\text { 2018b) }\end{array}$ \\
\hline $\begin{array}{l}\text { Arsenite (As } \\
\text { (III)) }\end{array}$ & $\begin{array}{l}\mathrm{Fe}^{2+}+\mathrm{NaS}_{2} \mathrm{O}_{4} \\
\quad \text { one-step }\end{array}$ & 0.21 & Anoxic & $\begin{array}{l}\text { Adsorption and } \\
\text { coprecipitation }\end{array}$ & 1.4 & -- & $\begin{array}{l}\text { Optimal at } \mathrm{pH} 7 \text {; reduced by } \mathrm{HCO}_{3}^{-} \text {, and } \\
\mathrm{HPO}_{4}{ }^{2-} ; \text { increased by DO }\end{array}$ & -- & (Wu et al., 2018) \\
\hline $\begin{array}{l}\text { Uranium (U } \\
(\mathrm{VI}))\end{array}$ & $\begin{array}{l}\text { nZVI + } \mathrm{Na}_{2} \mathrm{~S}, \\
\text { two-step }\end{array}$ & 0.5 & Anoxic & $\begin{array}{l}\text { Adsorption and } \\
\text { reductive } \\
\text { precipitation }\end{array}$ & 1.3 & $\mathrm{U}(\mathrm{IV})$ & $\begin{array}{c}\text { Increased with increasing } \mathrm{pH} ; \text { slightly } \\
\text { inhibited by } \mathrm{HA}, \mathrm{HCO}_{3}^{-} \text {, and } \mathrm{Ca}^{2+} ; \text { reduced } \\
\text { with aging }\end{array}$ & $\begin{array}{l}\text { Increased electrical } \\
\text { conductivity }\end{array}$ & $\begin{array}{l}\text { (Duan et al., } \\
\text { 2019) }\end{array}$ \\
\hline $\begin{array}{l}\text { Selenate (Se } \\
\text { (IV)) }\end{array}$ & $\begin{array}{l}\mathrm{mZVI}+\mathrm{S}^{0}, \text { ball } \\
\text { milling }\end{array}$ & 0.05 & Oxic & $\begin{array}{l}\text { Adsorption } \\
\text { followed by } \\
\text { reduction }\end{array}$ & 7.8 & Se $(0)$ & Reduced with increasing $\mathrm{pH}$ & $\begin{array}{c}\text { Increased anion } \\
\text { selectivity and electrical } \\
\text { conductivity }\end{array}$ & (Fan et al, 2019a) \\
\hline $\begin{array}{l}\text { Selenate (Se } \\
\text { (IV)) }\end{array}$ & $\begin{array}{l}\text { mZVI }+\mathrm{S}^{0} \text {, ball } \\
\text { milling }\end{array}$ & 0.05 & Oxic & $\begin{array}{l}\text { Adsorption } \\
\text { followed by } \\
\text { reduction }\end{array}$ & 194.0 & Se $(0)$ & Increased by $\mathrm{Fe}^{2+}$ & $\begin{array}{l}\text { Increased electrical } \\
\text { conductivity }\end{array}$ & (Fan et al, 2019b) \\
\hline $\begin{array}{l}\text { Chromate }(\mathrm{Cr} \\
\text { (VI)) }\end{array}$ & $\begin{array}{l}\mathrm{mZVI}+\mathrm{S}^{0} \text {, ball } \\
\text { milling }\end{array}$ & 0.05 & Oxic & Reduction & 3.7 & $\mathrm{Cr}$ (III) & -- & -- & $\begin{array}{l}\text { (Guan et al., } \\
\text { 2019) }\end{array}$ \\
\hline $\begin{array}{l}\text { Chromate (Cr } \\
\text { (VI)) }\end{array}$ & $\begin{array}{c}\mathrm{mZVI}+\mathrm{Na}_{2} \mathrm{~S}+ \\
\mathrm{Cu}^{2+}, \text { two-step }\end{array}$ & 0.056 & Oxic & $\begin{array}{l}\text { Reduction, } \\
\text { adsorption, and } \\
\text { precipitation }\end{array}$ & 2.4 & $\mathrm{Cr}$ (III) & Increased by $\mathrm{Cu}$ deposits & $\begin{array}{c}\text { Increased electrical } \\
\text { conductivity and release } \\
\text { of } \mathrm{Fe}(\mathrm{II})\end{array}$ & (Jia et al., 2019) \\
\hline $\begin{array}{l}\text { Selenate (Se } \\
(\mathrm{VI}))\end{array}$ & $\begin{array}{l}\mathrm{mZVI}+\mathrm{S}^{0} \text {, ball } \\
\text { milling }\end{array}$ & 0.05 & Oxic & $\begin{array}{l}\text { Adsorption and } \\
\text { reduction }\end{array}$ & $\sim 2$ & Se (0); Se (IV) & Reduced by $\mathrm{SO}_{4}{ }^{2-}, \mathrm{PO}_{4}{ }^{3-}$, and $\mathrm{Mg}^{2+}$ & -- & (Ling et al., 2019) \\
\hline $\begin{array}{c}\text { Chromate (Cr } \\
\text { (VI)) }\end{array}$ & $\begin{array}{c}\mathrm{Fe}^{3+}+\mathrm{NaS}_{2} \mathrm{O}_{4}, \\
\text { one-step }\end{array}$ & 0.5 & Anoxic & $\begin{array}{l}\text { Adsorption, } \\
\text { reduction, and } \\
\text { co-precipitation }\end{array}$ & 2.6 & $\left.\mathrm{Cr}(\mathrm{III}) ; \mathrm{Cr}_{\mathrm{x}} \mathrm{Fe}_{1-\mathrm{x}}\right)(\mathrm{OH})_{3}$ & Increased by increasing $\mathrm{pH}$ and IS & $\begin{array}{l}\text { Increased surface area, } \\
\text { electrical conductivity, } \\
\text { and }\end{array}$ & (Lv et al., 2019) \\
\hline
\end{tabular}




\begin{tabular}{|c|c|c|c|c|c|c|c|c|c|}
\hline $\begin{array}{l}\text { Uranium } \\
(\mathrm{U}(\mathrm{VI}))\end{array}$ & $\begin{array}{l}\mathrm{Fe}^{2+}+\mathrm{NaS}_{2} \mathrm{O}_{4} \\
\text { one-step }\end{array}$ & 0.14 & Anoxic & $\begin{array}{l}\text { Adsorption and } \\
\text { reduction }\end{array}$ & 1.2 & -- & Increased by deposits on biochar & -- & (Pang et al., 2019) \\
\hline $\begin{array}{l}\text { Chromate (Cr } \\
\text { (VI)) }\end{array}$ & $\begin{array}{c}\mathrm{nZVI}+\mathrm{Na}_{2} \mathrm{~S} \\
\text { two-step; } \mathrm{Fe}^{2+}+ \\
\mathrm{Na}_{2} \mathrm{~S}, \text { one-step; }\end{array}$ & 0.35 & Anoxic & $\begin{array}{l}\text { Adsorption and } \\
\text { reduction }\end{array}$ & 1.3 & $\mathrm{Cr}$ (III) & $\begin{array}{l}\text { Reduced by increasing } \mathrm{pH} \text {, aging, and in } \\
\mathrm{GW}\end{array}$ & -- & (Zhao et al., 2019) \\
\hline $\begin{array}{l}\text { Chromate (Cr } \\
\text { (VI) }\end{array}$ & $\begin{array}{c}\mathrm{mZVI}+\mathrm{S}^{0}, \text { ball } \\
\text { milling }\end{array}$ & 0.2 & Anoxic & Chemisorption & 10.7 & $\begin{array}{c}\left(\mathrm{Cr}_{\mathrm{x}} \mathrm{Fe}_{1-x}\right)(\mathrm{OH})_{3} ; \\
\text { formed a passivation } \\
\text { layer }\end{array}$ & Reduced by increasing $\mathrm{pH}$ and by DO & Increased surface area & (Zou et al., 2019) \\
\hline $\begin{array}{l}\text { Arsenite (As } \\
\text { (III)) }\end{array}$ & $\begin{array}{l}\text { mZVI }+\mathrm{FeS}_{2} \\
\text { ball milling }\end{array}$ & 1 & Oxic & $\begin{array}{l}\text { Adsorption, co- } \\
\text { precipitation, } \\
\text { and oxidation }\end{array}$ & 5.0 & As $(\mathrm{V})$ & Reduced with increasing $\mathrm{pH}$ & $\begin{array}{l}\text { Increased surface area and } \\
\text { electrical conductivity }\end{array}$ & (Du et al., 2020) \\
\hline $\begin{array}{l}\text { Chromate } \\
\left(\mathrm{Cd}^{2+}\right)\end{array}$ & $\begin{array}{l}\mathrm{Fe}^{3+}+\mathrm{Na}_{2} \mathrm{~S} \\
\text { one-step }\end{array}$ & 0.75 & Anoxic & $\begin{array}{l}\text { Replacement of } \\
\text { FeS by CdS }\end{array}$ & 2.8 & $\mathrm{CdS}$ & Slightly reduced by $\mathrm{NO}_{3}^{-}, \mathrm{Mg}^{2+}$, and $\mathrm{HA}$ & -- & $\begin{array}{l}\text { (Liang et al., } \\
2020)\end{array}$ \\
\hline $\begin{array}{l}\text { Copper } \\
\left(\mathrm{Cu}^{2+}\right)\end{array}$ & $\begin{array}{l}\mathrm{Fe}^{3+}+\mathrm{NaS}_{2} \mathrm{O}_{4} \\
\text { one-step }\end{array}$ & 0.1 & Anoxic & Reduction & 1.4 & n.a. & $\begin{array}{c}\text { Increased with } \mathrm{pH} \text {; decreased by } \mathrm{Cl}^{-}, \mathrm{NO}_{3}^{-} \text {, } \\
\text { and } \mathrm{HA}\end{array}$ & -- & (Xu et al., 2020e) \\
\hline Nickel $\left(\mathrm{Ni}^{2+}\right)$ & $\begin{array}{c}\mathrm{Fe}^{3+}+\mathrm{NaS}_{2} \mathrm{O}_{4} \\
\text { one-step }\end{array}$ & 0.1 & Anoxic & Sorption & 3.1 & n.a. & Not affected by $\mathrm{pH}, \mathrm{Cl}^{-}, \mathrm{NO}_{3}^{-}$, and $\mathrm{HA}$ & -- & \\
\hline
\end{tabular}

* The proposed mechanism for enhanced reactivity of S-(n)ZVI to (n)ZVI.

n.a. not available; HA, humic acid; GW, groundwater; IS, ionic strength; OM, organic matter; DO, dissolved oxygen; PDS, peroxydisulfate; PMS, peroxymonosulfate. 

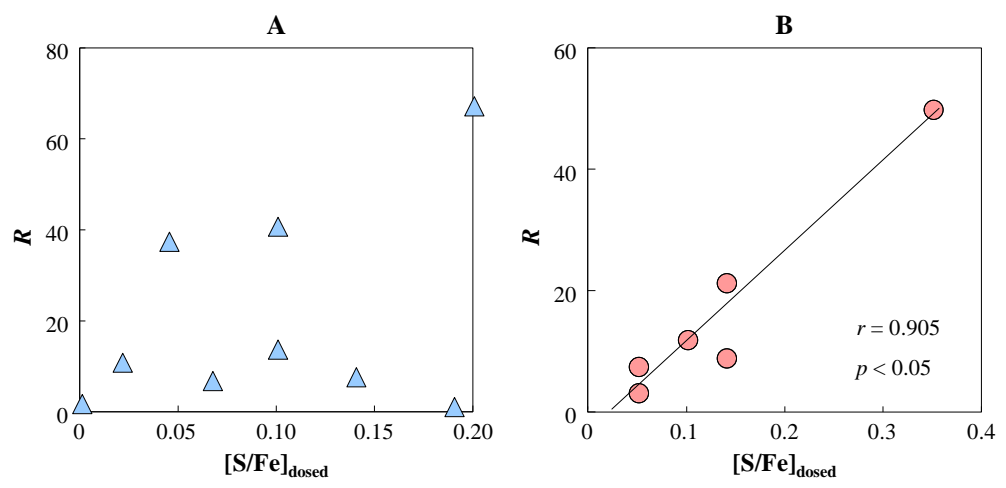

Figure S2. Correlation of the enhancement ratio $(R)$ in reactivity of TCE induced by sulfidation of (n)ZVI with the $[\mathrm{S} / \mathrm{Fe}]_{\text {dosed }}$ of S-(n)ZVI synthesized by (A) two-step method with $\mathrm{Na}_{2} \mathrm{~S}$ and (B) one-step method with $\mathrm{Na}_{2} \mathrm{~S}_{2} \mathrm{O}_{4}$. The correlation coefficient $(r)$ and the significance level $(p)$ are shown. 


\section{References}

(1) Hassan, S. M. Reduction of halogenated hydrocarbons in aqueous media: I. Involvement of sulfur in iron catalysis. Chemosphere 2000, 40 (12), 1357-1363.

(2) Butler, E. C.; Hayes, K. F. Factors influencing rates and products in the transformation of trichloroethylene by iron sulfide and iron metal. Environ. Sci. Technol. 2001, 35 (19), 3884-3891.

(3) Kim, E. J.; Kim, J. H.; Azad, A. M.; Chang, Y. S. Facile synthesis and characterization of Fe/FeS nanoparticles for environmental applications. ACS Appl. Mater. Interface, 2011, 3 (5), 1457-1462.

(4) Kim, E. J.; Murugesan, K.; Kim, J. H.; Tratnyek, P. G.; Chang, Y. S. Remediation of trichloroethylene by FeS-coated iron nanoparticles in simulated and real groundwater: Effects of water chemistry. Ind. Eng. Chem. Res. 2013, 52 (27), 9343-9350.

(5) Kim, E. J.; Kim, J. H.; Chang, Y. S.; Turcio-Ortega, D.; Tratnyek, P. G. Effects of metal ions on the reactivity and corrosion electrochemistry of Fe/FeS nanoparticles. Environ. Sci. Technol. 2014, 48 (7), 4002-4011.

(6) Rajajayavel, S. R. C.; Ghoshal, S. Enhanced reductive dechlorination of trichloroethylene by sulfidated nanoscale zerovalent iron. Water Res. 2015, 78, 144-153.

(7) Han, Y.; Yan, W. Reductive Dechlorination of trichloroethene by zero-valent iron nanoparticles: Reactivity enhancement through sulfidation treatment. Environ. Sci. Technol. 2016, 50 (23), 1299213001.

(8) Fan, D.; O’Brien Johnson, G.; Tratnyek, P. G.; Johnson, R. L. Sulfidation of nano zerovalent iron (nZVI) for improved selectivity during in-situ chemical reduction (ISCR). Environ. Sci. Technol. 2016, 50 (17), 9558-9565.

(9) Gu, Y.; Wang, B.; He, F.; Bradley, M. J.; Tratnyek, P. G. Mechanochemically sulfidated microscale zero valent iron: Pathways, kinetics, mechanism, and efficiency of trichloroethylene dechlorination. Environ. Sci. Technol. 2017, 51, 12653-12662.

(10) He, F.; Li, Z; Shi, S.; Xu, W.; Sheng, H.; Gu, Y.; Jiang, Y.; Xi, B. Dechlorination of excess trichloroethene by bimetallic and sulfidated nanoscale zero-valent iron. Environ. Sci. Technol. 2018, 52: $8627-8637$.

(11) Bhattacharjee, S.; Ghoshal, S. Sulfidation of nanoscale zerovalent iron in the presence of two organic macromolecules and its effects on trichloroethene degradation. Environ. Sci. Nano 2018a, 5 (3), $782-$ 791.

(12) Bhattacharjee, S.; Ghoshal, S. Optimal design of sulfidated nanoscale zerovalent iron for enhanced trichloroethene degradation. Environ. Sci. Technol. 2018b, 52(19), 11078-11086.

(13) Dong, H.; Zhang, C.; Deng, J.; Jiang, Z.; Zhang, L.; Cheng, Y.; Hou, K.; Tang, L.; Zeng, G. Factors influencing degradation of trichloroethylene by sulfide-modified nanoscale zero-valent iron in aqueous solution. Water Res. 2018, 135, 1-10.

(14) Mangayayam, M.; Dideriksen, K.; Ceccato, M.; Tobler, D. J. The structure of sulfidized zero valent iron by one-step synthesis: Impact on contaminant selectivity and long-term performance. Environ. Sci. Technol., 2019a, 53 (8), 4389-4396.

(15) Mangayayam, M. C.; Perez, J. P. H.; Dideriksen, K.; Freeman, H. M.; Bovet, N.; Benning, L. G.; Tobler, D. J. Structural transformation of sulfidized zerovalent iron and its impact on long-term reactivity. Environ. Sci. Nano 2019, 6 (11), 3422-3430. 
(16) Mangayayam, M. C.; Alonso-de-Linaje, V.; Dideriksen, K.; Tobler, D. J. Effects of common groundwater ions on the transformation and reactivity of sulfidized nanoscale zerovalent iron. Chemosphere 2020, 249, 126137.

(17) Xu, J.; Wang, Y.; Weng, C.; Bai, W.; Jiao, Y.; Kaegi, R.; Lowry, G. V. Reactivity, Selectivity, and Long-term Performance of Sulfidized Nanoscale Zerovalent Iron with Different Properties. Environ. Sci. Technol. 2019, 53, 5936-5945.

(18) Xu, J.; Cao, Z.; Zhou, H.; Lou, Z.; Wang, Y.; Xu, X.; Lowry, G. V. Sulfur Dose and Sulfidation Time Affect Reactivity and Selectivity of Two-step-Sulfidized Nanoscale Zerovalent Iron. Environ. Sci. Technol. 2019. 53 (22), 13344-13352.

(19) Gu, Y.; Gong, Li.; Qi, J.; Cai, S.; Tu, W.; He, F. Sulfidation mitigates the passivation of zero valent iron at alkaline pHs: Experimental evidences and mechanism. Water Res. 2019, 159: 233-241.

(20) Zou, H.; Hu, E.; Yang, S.; Gong, L.; He, F. Chromium (VI) removal by mechanochemically sulfidated zero valent iron and its effect on dechlorination of trichloroethene as a co-contaminant. Sci. Total. Environ. 2019, 650, 419-426.

(21) Han, Y.; Ghoshal, S.; Lowry, G. V.; Chen, J. A comparison of the effects of natural organic matter on sulfidated and nonsulfidated nanoscale zerovalent iron colloidal stability, toxicity, and reactivity to trichloroethylene. Sci. Total Environ. 2019, 671, 254-261.

(22) Islam, S.; Han, Y.; Yan, W. Reactions of chlorinated ethenes with surface-sulfidated iron materials: reactivity enhancement and inhibition effects. Environ. Sci.: Process. Impacts 2020.

(23) Wang, B.; Dong, H.; Li, L.; Wang, Y.; Ning, Q.; Tang, L.; Zeng, G. Influence of different cocontaminants on trichloroethylene removal by sulfide-modified nanoscale zero-valent iron. Chem. Eng. J. 2020, 381, 122773.

(24) Xu, W.; Li, Z.; Shi, S.; Qi, J.; Cai, S.; Yu, Y.; O'Carroll, D. M.; He, F. Carboxymethyl cellulose stabilized and sulfidated nanoscale zero-valent iron: Characterization and trichloroethene dechlorination. Appl. Catal. 2020, 262, 118303.

(25) Bin, Q.; Lin, B.; Zhu, K.; Shen, Y.; Man, Y.; Wang, B.; Lai, C.; Chen, W. Superior trichloroethylene removal from water by sulfide-modified nanoscale zero-valent iron/graphene aerogel composite. J. Environ. Sci. 88, 2020, 90-102.

(26) Xu, B. D.; Li, D. C.; Qian, T. T.; Jiang, H. Boosting the activity and environmental stability of nanoscale zero-valent iron by montmorillonite supporting and sulfidation treatment. Chem. Eng. J. 2020a, 124063.

(27) Xu, J.; Avellan, A.; Li, H.; Liu, X.; Noël, V.; Lou, Z.; Wang, Y.; Kaegi, R.; Henkelman, G.; Lowry, G. V. Sulfur Loading and Speciation Control the Hydrophobicity, Electron Transfer, Reactivity, and Selectivity of Sulfidized Nanoscale Zerovalent Iron. Adv. Mater. 2020b, 1906910.

(28) Brumovský, M.; Filip, J.; Malina, O.; Oborná, J.; Sracek, O.; Reichenauer, T. G.; Andrýsková, P.; Zbořil, R. Core-shell $\mathrm{Fe} / \mathrm{FeS}$ nanoparticles with controlled shell thickness for enhanced trichloroethylene removal. ACS Appl. Mater. Interfaces 2020. 12 (31), 35424-35434.

(29) Gong, L.; Lv, N.; Qi, J.; Qiu, X.; Gu, Y.; He, F. Effects of non-reducible dissolved solutes on reductive dechlorination of trichloroethylene by ball milled zero valent irons. J. Hazard. Mater. 2020, 122620.

(30) Cai, S.; Chen, B.; Qiu, X.; Li, J.; Tratnyek, P. G.; He, F. Sulfidation of zero-valent iron by direct reaction with elemental sulfur in water: Efficiencies, mechanism, and dechlorination of trichloroethylene. Environ. Sci. Technol. 2021. 
(31) Gong, L.; Qi, J.; Lv, N.; Qiu, X.; Gu, Y.; Zhao, J.; He, F. Mechanistic role of nitrate anion in TCE dechlorination by ball milled ZVI and sulfidated ZVI: Experimental investigation and theoretical analysis. J. Hazard. Mater. 2021, 403: 123844.

(32) Xu, J.; Avellan, A.; Li, H.; Clark, E. A.; Henkelman, G.; Kaegi, R.; Lowry, G. V. Iron and sulfur precursors affect crystalline structure, speciation, and reactivity of sulfidized nanoscale zerovalent iron. Environ. Sci. Technol. 2020d, 54 (20), 13294-13303.

(33) Nezamabadi, S.G. Accelerated Degradation of Chlorinated Solvents by Nanoscale Zero Valent Iron Coated with Iron Monosolfide and Stabilized with Carboxymethyl Cellulose. Diss. Wright State University, 2016.

(34) Vogel, M.; Georgi, A.; Kopinke, F. D.; Mackenzie, K. Sulfidation of ZVI/AC composite leads to highly corrosion-resistant nanoremediation particles with extended life-time. Sci. Total Environ. 2019, $665,235-245$.

(35) Nunez Garcia, A.; Boparai, H. K.; O'Carroll, D. M. Enhanced dechlorination of 1, 2-dichloroethane by coupled nano iron-dithionite treatment. Environ. Sci. Technol. 2016, 50(10), 5243-5251.

(36) Jin, X.; Chen, H.; Yang, Q.; Hu, Y.; Yang, Z. Dechlorination of carbon tetrachloride by sulfidemodified nanoscale zerovalent iron. Environ. Eng. 201835 (6), 560-567.

(37) Tang, J.; Tang, L.; Feng, H.; Zeng, G.; Dong, H.; Zhang, C.; Huang, B.; Deng, Y.; Wang, J.; Zhou, Y. pH-dependent degradation of $\mathrm{p}$-nitrophenol by sulfidated nanoscale zerovalent iron under aerobic or anoxic conditions. J. Hazard. Mater. 2016, 320, 581-590.

(38) Li, D.; Mao, Z.; Zhong, Y.; Huang, W.; Wu, Y. Reductive transformation of tetrabromobisphenol A by sulfidated nano zerovalent iron. Water research, 2016, 103, 1-9.

(39) Li, D.; Zhu, X.; Zhong, Y.; Huang, W. Abiotic transformation of hexabromocyclododecane by sulfidated nanoscale zerovalent iron: Kinetics, mechanism and influencing factors. Water Res. 2017, $121,140-149$.

(40) Cao, Z.; Liu, X.; Xu, J.; Zhang, J.; Yang, Y.; Zhou, J. L.; Xu, X. H.; Lowry, G. V. Removal of Antibiotic Florfenicol by Sulfide-Modified Nanoscale Zero-Valent Iron. Environ. Sci. Technol. 2017, 51, 11269-11277.

(41) Zhou, G. N.; Li, W. Q.; He, C. S.; Liu, X. C.; Ding, R. R.; Wang, Y. X.; Mu, Y. Enhanced hydrodeiodination of iodinated contrast medium by sulfide-modified nano-sized zero-valent iron: Kinetics, mechanisms and application prospects. Chem. Eng. J. 2020, 401, 126050.

(42) Wang, S.; Song, Y.; Sun, Y. Enhanced dyes removal by sulfidated zerovalent iron: Kinetics and influencing factors. Environ.Technol. Inno. 2018, 11, 339-347.

(43) Wei, X.; Yin, H.; Peng, H.; Chen, R.; Lu, G.; Dang, Z. Reductive debromination of decabromodiphenyl ether by iron sulfide-coated nanoscale zerovalent iron: mechanistic insights from Fe (II) dissolution and solvent kinetic isotope effects. Environ Pollut. 2019, 253, 161-170.

(44) Qin, H.; Guan, X.; Tratnyek, P. G. Effects of sulfidation and nitrate on the reduction of Nnitrosodimethylamine by zerovalent iron. Environ. Sci. Technol. 2019, 53 (16), 9744-9754.

(45) Lou, Y.; Cai, Y.; Tong, Y.; Hsieh, L.; Li, X.; Xu, W.; Shi, K.; Shen, C.; Xu, X.; Lou, L.Interaction between pollutants during the removal of polychlorinated biphenyl-heavy metal combined pollution by modified nanoscale zero-valent iron. Sci. Total Environ. 2019, 673, 120-127.

(46) Zhang, D.; Li, Y.; Sun, A.; Tong, S.; Jiang, X.; Mu, Y.; Li, J.; Han, W.; Sun, X.; Wang, L.; Shen, J. Optimization of $\mathrm{S} / \mathrm{Fe}$ ratio for enhanced nitrobenzene biological removal in anaerobicSystem amended withSulfide-modified nanoscale zerovalent iron. Chemosphere 2020, 125832. 
(47) Cao, Z.; Li, H.; Xu, X.; Xu, J. Correlating surface chemistry and hydrophobicity of sulfidized nanoscale zerovalent iron with its reactivity and selectivity for denitration and dechlorination. Chem. Engineer. J. 2020a, 124876.

(48) Cao, Z.; Xu, J.; Li, H.; Ma, T.; Lou, L.; Henkelman, G.; Xu, X. Dechlorination and defluorination capability of sulfidized nanoscale zerovalent iron with suppressed water reactivity. Chem. Engineer. J. 2020b, 400, 125900.

(49) Tian, X.; Wang, X.; Nie, Y.; Yang, C.; Dionysiou, D. D. Hyroxyl radical-involving p-nitrophenol oxidation during its reduction by nanoscale sulfidated zerovalent iron under anaerobic conditions. Environ. Sci. Technol. 2021, 55, 2403-2410.

(50) Zhang, D.; Li, Y.; Tong, S.; Jiang, X.; Wang, L.; Sun, X.; Li, J.; Liu, X.; Shen, J. Biochar supported sulfide-modified nanoscale zero-valent iron for the reduction of nitrobenzene. RSC Adv. 2018, 8 (39), 22161-22168.

(51) Yang, L.; Gao, J.; Liu, Y.; Zhang, Z.; Zou, M.; Liao, Q.; Shang, J. Removal of Methyl Orange from Water Using Sulfur-Modified nZVI Supported on Biochar Composite. Water Air Soil Pollut. 2018, 229 (11), 355 .

(52) Zhang, D.; Li, Y.; Sun, A.; Tong, S.; Su, G.; Jiang, X.; Li, J.; Han, W.; Sun, X.; Wang, L.; Shen, J. Enhanced nitrobenzene reduction by modified biochar supported sulfidated nano zerovalent iron: Comparison of surface modification methods. Sci. Total Environ. 2019, 694, 133701.

(53) Xu, J.; Cao, Z.; Wang, Y.; Zhang, Y.; Gao, X.; Ahmed, M. B.; Zhang, J.; Yang, Y.; Zhou, J. L.; Lowry, G. V. Distributing sulfidized nanoscale zerovalent iron onto phosphorus-functionalized biochar for enhanced removal of antibiotic florfenicol. Chem. Eng. J. 2019d, 359, 713-722.

(54) Lu, J.; Zhang, C.; Wu, J. One-step synthesis of magnetic algal carbon/sulfidated nanoscale zerovalent iron composites for removal of bromated disinfection by-product. Chemosphere 2020, 126257.

(55) Cao, Z.; Li, H.; Lowry, G. V.; Shi, X.; Pan, X.; Xu, X.; Henkelman, G.; Xu, J. Unveiling the role of sulfur in rapid defluorination of florfenicol by sulfidized nanoscale zero-valent iron in water under ambient conditions. Environ. Sci. Technol. 2021, 55 (4), 2628-2638.

(56) Lipczynska-Kochany, E.; Harms, S.; Milburn, R.; Sprah, G.; Nadarajah, N. Degradation of carbon tetrachloride in the presence of iron and sulphur containing compounds. Chemosphere 1994, 29 (7), 1477-1489.

(57) Du, J.; Bao, J.; Fu, X.; Lu, C.; Kim, S. H. Mesoporous sulfur-modified iron oxide as an effective Fenton-like catalyst for degradation of bisphenol A. Appl. Catal. B-Environ. 2016, 184, 132-141.

(58) Tang, J.; Tang, L.; Feng, H.; Zeng, G.; Dong, H.; Zhang, C.; Huang, B.; Deng, Y.; Wang, J.; Zhou, Y. $\mathrm{pH}$-dependent degradation of $\mathrm{p}$-nitrophenol by sulfidated nanoscale zerovalent iron under aerobic or anoxic conditions. J. Hazard. Mater. 2016, 320, 581-590.

(59) Xu, C.; Zhang, B.; Wang, Y.; Shao, Q.; Zhou, W.; Fan, D.; Bandstra, J. Z.; Shi, Z. Q.; Tratnyek, P. G. Effects of sulfidation, magnetization, and oxygenation on azo dye reduction by zerovalent iron. Environ. Sci. Technol. 2016, 50 (21), 11879-11887.

(60) Zhang, Q.; Guo, W.; Yue, X.; Liu, Z.; Li, X. Degradation of rhodamine B using FeS- coated zerovalent iron nanoparticles in the presence of dissolved oxygen. Environ. Prog. Sustain. Energy 2016, 35 (6), 1673-1678.

(61) Song, S.; Su, Y.; Adeleye, A. S.; Zhang, Y.; Zhou, X. Optimal design and characterization of sulfidemodified nanoscale zerovalent iron for diclofenac removal. Appl. Catal. 2017, 201, 211-220. 
(62) Rayaroth, M. P.; Lee, C. S.; Aravind, U. K.; Aravindakumar, C. T.; Chang, Y. S. Oxidative degradation of benzoic acid using $\mathrm{Fe} 0$-and sulfidized $\mathrm{Fe} 0$-activated persulfate: a comparative study. Chem. Eng. J. 2017, 315, 426-436.

(63) Su, Y.; Jassby, D.; Song, S.; Zhou, X.; Zhao, H.; Filip, J.; Petala, E.; Zhang, Y. Enhanced oxidative and adsorptive removal of diclofenac in heterogeneous Fenton-like reaction with sulfide modified nanoscale zerovalent iron. Environ. Sci. Technol. 2018, 52 (11), 6466-6475.

(64) Wu, J.; Zhao, J.; Hou, J.; Zeng, R. J.; Xing, B. Degradation of tetrabromobisphenol a by sulfidated nanoscale zerovalent iron in a dynamic two-step anoxic/oxic process. Environ. Sci. Technol. 2019, 53 (14), 8105-8114.

(65) Zhang, L.; Shao, Q.; Xu, C. Enhanced azo dye removal from wastewater by coupling sulfidated zerovalent iron with a chelator. J. Clean. Prod. 2019. 213, 753-761.

(66) Dong, H.; Hou, K.; Qiao, W.; Cheng, Y.; Zhang, L.; Wang, B.; Li, L.; Wang, Y.; Ning, Q.; Zeng, G. Insights into enhanced removal of TCE utilizing sulfide-modified nanoscale zero-valent iron activated persulfate. Chem. Eng. J. 2019a, 359, 1046-1055.

(67) Dong, H.; Wang, B.; Li, L.; Wang, Y.; Ning, Q.; Tian, R.; Rui, L.; Chen, J.; Xie, Q. Activation of persulfate and hydrogen peroxide by using sulfide-modified nanoscale zero-valent iron for oxidative degradation of sulfamethazine: A comparative study. Sep. Purif. Technol. 2019b, 218, 113-119.

(68) Li, Y.; Zhao, X.; Yan, Y.; Yan, J.; Pan, Y.; Zhang, Y.; Lai, B. Enhanced sulfamethoxazole degradation by peroxymonosulfate activation with sulfide-modified microscale zero-valent iron (S$\mathrm{mFe}$ ): Performance, mechanisms, and the role of sulfur species. Chem. Eng. J. 2019, 376, 2019, 21302.

(69) Guo, W.; Zhao, Q.; Du, J.; Wang, H.; Li, X.; Ren, N. Enhanced removal of sulfadiazine by sulfidated ZVI activated persulfate process: Performance, mechanisms and degradation pathways. Chem. Eng. J. 2020, 388, 124303.

(70) Rayaroth, M. P.; Prasanthkumar, K. P.; Kang, Y. G.; Lee, C. S.; Chang, Y. S. Degradation of carbamazepine by singlet oxygen from sulfidized nanoscale zero-valent iron-citric acid system. Chem. Eng. J. 2020, 382, 122828.

(71) Qiao, J.; Guo, Y.; Dong, H.; Guan, X.; Zhou, G.; Sun, Y. Activated peroxydisulfate by sulfidated zero-valent iron for enhanced organic micropollutants removal from water. Chem. Eng. J. 2020, 125301.

(72) Li, R.; Dong, H. R.; Tian, R.; Chen, J.; Xie, Q. Q. Activation of sulfite by different Fe0-based nanomaterials for oxidative removal of sulfamethazine in aqueous solution. Sep. Purif. Technol. 2020, 117230.

(73) Fan, D.; Anitori, R. P.; Tebo, B. M.; Tratnyek, P. G.; Lezama Pacheco, J. S.; Kukkadapu, R. K.; Arey, B. W. Reductive sequestration of pertechnetate (99TcO4-) by nano zerovalent iron (nZVI) transformed by abiotic sulfide. Environ. Sci. Technol. 2013, 47(10), 5302-5310.

(74) Su, Y.; Adeleye, A. S.; Keller, A. A.; Huang, Y.; Dai, C.; Zhou, X.; Zhang, Y., Magnetic sulfidemodified nanoscale zerovalent iron (S-nZVI) for dissolved metal ion removal. Water Res. 2015, 74, 47-57.

(75) Su, Y.; Adeleye, A. S.; Huang, Y.; Zhou, X.; Keller, A. A.; Zhang, Y. Direct synthesis of novel and reactive sulfide-modified nano iron through nanoparticle seeding for improved cadmium-contaminated water treatment. Sci. Rep. 2016, 6, 24358.

(76) Du, J. K.; Bao, J. G.; Lu, C. H.; Werner, D., Reductive sequestration of chromate by hierarchical FeS@Fe-0 particles. Water research 2016, 102, 73-81. 
(77) Gong, Y.; Gai, L.; Tang, J.; Fu, J.; Wang, Q.; Zeng, E. Y. Reduction of Cr (VI) in simulated groundwater by FeS-coated iron magnetic nanoparticles. Sci. Total. Environ. 2017, 595, 743-751.

(78) Gao, J.; Yang, L.; Liu, Y.; Shao, F.; Liao, Q.; Shang, J., Scavenging of Cr(VI) from aqueous solutions by sulfide-modified nanoscale zero-valent iron supported by biochar. Journal of the Taiwan Institute of Chemical Engineers 2018. 91, 449-456.

(79) Huang, S.; Xu, C.; Shao, Q.; Wang, Y.; Zhang, B.; Gao, B.; Zhou, W.; Tratnyek, P. G., Sulfidemodified zerovalent iron for enhanced antimonite sequestration: Characterization, performance, and reaction mechanisms. Chem Eng J 2018, 338, 539-547.

(80) Li, J.; Zhang, X.; Liu, M.; Pan, B.; Zhang, W.; Shi, Z.; Guan, X. Enhanced reactivity and electron selectivity of sulfidated zerovalent iron toward chromate under aerobic conditions. Environ. Sci. Technol. 2018, 52(5), 2988-2997.

(81) Lv, D.; Zhou, X.; Zhou, J.; Liu, Y.; Li, Y.; Yang, K.; Lou, Z.; Baig, S. A.; Wu, D.; Xu, X., Design and characterization of sulfide-modified nanoscale zerovalent iron for cadmium(II) removal from aqueous solutions. Applied Surface Science 2018, 442, 114-123.

(82) Shao, Q.; Xu, C.; Wang, Y.; Huang, S.; Zhang, B.; Huang, L.; Fan, D.; Tratnyek, P. G., Dynamic interactions between sulfidated zerovalent iron and dissolved oxygen: Mechanistic insights for enhanced chromate removal. Water research 2018, 135, 322-330.

(83) Wang, Y.; Shao, Q.; Huang, S.; Zhang, B.; Xu, C., High performance and simultaneous sequestration of $\mathrm{Cr}(\mathrm{VI})$ and $\mathrm{Sb}(\mathrm{III})$ by sulfidated zerovalent iron. J Clean Prod 2018, 191, 436-444.

(84) Wu, D.; Peng, S.; Yan, K.; Shao, B.; Feng, Y.; Zhang, Y., Enhanced As(III) sequestration using sulfide-modified nanoscale zerovalent iron with a characteristic core-shell structure: Sulfidation and As distribution. ACS Sustainable Chemistry \& Engineering 2018, 6, 3039-3048.

(85) Duan, J.; Ji, H.; Liu, W.; Zhao, X.; Han, B.; Tian, S.; Zhao, D., Enhanced immobilization of U(VI) using a new type of FeS-modified Fe0 core-shell particles. Chem. Eng. J. 2019, 359, 1617-1628.

(86) Fan, P.; Li, L.; Sun, Y.; Qiao, J.; Xu, C.; Guan, X. Selenate removal by Fe0 coupled with ferrous iron, hydrogen peroxide, sulfidation, and weak magnetic field: A comparative study. Water Res. 2019a, $159,375-384$.

(87) Fan, P.; Sun, Y.; Zhou, B.; Guan, X. Coupled Effect of Sulfidation and Ferrous Dosing on Selenate Removal by Zerovalent Iron Under Aerobic Conditions. Environ. Sci. Technol 2019b, 53 (24), 14577-14585.

(88) Guan, X.; Yang, H.; Sun, Y.; Qiao, J. Enhanced immobilization of chromium (VI) in soil using sulfidated zero-valent iron. Chemosphere 2019, 228, 370-376.

(89) Jia, T.; Zhang, B.; Huang, L.; Wang, S.; Xu, C., Enhanced sequestration of Cr(VI) by copper doped sulfidated zerovalent iron (SZVI-Cu): Characterization, performance, and mechanisms. Chem Eng J 2019, 366, 200-207.

(90) Ling, J.; Qiao, J.; Song, Y.; Sun, Y. Influence of coexisting ions on the electron efficiency of sulfidated zerovalent iron toward Se (VI) removal. Chem. Eng. J. 2019, 378, 122124.

(91) Lv, D.; Zhou, J.; Cao, Z.; Xu, J.; Liu, Y.; Li, Y.; Xu, X. Mechanism and influence factors of chromium (VI) removal by sulfide-modified nanoscale zerovalent iron. Chemosphere. 2019, 224, $306-315$.

(92) Pang, H.; Diao, Z.; Wang, X.; Ma, Y.; Yu, S.; Zhu, H.; Wang, X. Adsorptive and reductive removal of U (VI) by Dictyophora indusiate-derived biochar supported sulfide NZVI from wastewater. Chem. Eng. J. 2019, 366, 368-377. 
(93) Zhao, L.; Zhao, Y.; Yang, B.; Teng, H. Application of carboxymethyl Cellulose-stabilized sulfidated nano zerovalent iron for removal of Cr (VI) in simulated groundwater. Water Air Soil Pollut. 2019, 230 (6), 113.

(94) Zou, H.; Hu, E.; Yang, S.; Gong, L.; He, F. Chromium (VI) removal by mechanochemically sulfidated zero valent iron and its effect on dechlorination of trichloroethene as a co-contaminant. Sci. Total Environ. 2019, 650, 419-426.

(95) Du, M.; Zhang, Y.; Zeng, X.; Kuang, H.; Huang, S. Enhancement of ball-miling on pyrite/zero-valent iron for arsenic removal in water: A mechanistic study. Chemosphere 2020. 249, 126130.

(96) Liang, L.; Li, X.; Lin, Z.; Tian, C.; Guo, Y. The removal of Cd by sulfidated nanoscale zero-valent iron: The structural, chemical bonding evolution and the reaction kinetics. Chem. Eng. J. 2020, 382, 122933.

(97) Xu, W.; Hu, X.; Lou, Y.; Jiang, X.; Shi, K.; Tong, Y.; Xu, X.; Shen, C.; Hu, B.; Lou, L. Effects of environmental factors on the removal of heavy metals by sulfide-modified nanoscale zerovalent iron. Environ. Res. 2020, 109662. 